\title{
Cell Growth Rate Dictates the Onset of Glass to Fluidlike Transition and Long Time Superdiffusion in an Evolving Cell Colony
}

\author{
Abdul N. Malmi-Kakkada, ${ }^{1}$ Xin Li,${ }^{1}$ Himadri S. Samanta, ${ }^{1}$ Sumit Sinha, ${ }^{2}$ and D. Thirumalai ${ }^{1, *}$ \\ ${ }^{1}$ Department of Chemistry, University of Texas, Austin, Texas 78712, USA \\ ${ }^{2}$ Department of Physics, University of Texas, Austin, Texas 78712, USA
}

(Received 7 August 2017; revised manuscript received 13 February 2018; published 27 April 2018)

Collective migration dominates many phenomena, from cell movement in living systems to abiotic selfpropelling particles. Focusing on the early stages of tumor evolution, we enunciate the principles involved in cell dynamics and highlight their implications in understanding similar behavior in seemingly unrelated soft glassy materials and possibly chemokine-induced migration of $\mathrm{CD} 8^{+} \mathrm{T}$ cells. We performed simulations of tumor invasion using a minimal three-dimensional model, accounting for cell elasticity and adhesive cell-cell interactions, as well as cell birth and death, to establish that cell-growth-ratedependent tumor expansion results in the emergence of distinct topological niches. Cells at the periphery move with higher velocity perpendicular to the tumor boundary, while the motion of interior cells is slower and isotropic. The mean-square displacement $\Delta(t)$ of cells exhibits glassy behavior at times comparable to the cell cycle time, while exhibiting superdiffusive behavior, $\Delta(t) \approx t^{\alpha}(\alpha>1)$, at longer times. We derive the value of $\alpha \approx 1.33$ using a field theoretic approach based on stochastic quantization. In the process, we establish the universality of superdiffusion in a class of seemingly unrelated nonequilibrium systems. Superdiffusion at long times arises only if there is an imbalance between cell birth and death rates. Our findings for the collective migration, which also suggest that tumor evolution occurs in a polarized manner, are in quantitative agreement with in vitro experiments. Although set in the context of tumor invasion, the findings should also hold in describing the collective motion in growing cells and in active systems, where creation and annihilation of particles play a role.

DOI: 10.1103/PhysRevX.8.021025

\author{
Subject Areas: Biological Physics, \\ Computational Physics, \\ Interdisciplinary Physics, Soft Matter, \\ Statistical Physics
}

\section{INTRODUCTION}

The strict control of cell division and apoptosis is critical for tissue development and maintenance [1]. Dysfunctional cell birth and death control mechanisms lead to several physiological diseases, including cancers [2]. Together with genetic cues controlling birth-death processes, mechanical behavior of a collection of cells is thought to be of fundamental importance in biological processes such as embryogenesis, wound healing, stem cell dynamics, morphogenesis, tumorigenesis, and metastasis [3-8]. Because of the interplay between birth-death processes and cell-cell interactions, we expect that the collective motion of cells ought to exhibit unusual nonequilibrium dynamics, whose

\footnotetext{
*dave.thirumalai@gmail.com
}

Published by the American Physical Society under the terms of the Creative Commons Attribution 4.0 International license. Further distribution of this work must maintain attribution to the author(s) and the published article's title, journal citation, and DOI. understanding might hold the key to describing tumor invasion and related phenomena. Interestingly, characteristics of glasslike behavior such as diminished motion (jamming) of a given cell in a dense environment created by neighboring cells (caging effect), dynamic heterogeneity, and possible viscoelastic response have been reported in confluent tissues $[9,10]$. Using imaging techniques that track cell motions, it has been shown that in both two (kidney cells on a flat, thick polyacrylamide gel $[9,11]$ ) and three dimensions (explants from zebrafish embedded in agarose [12]) the mean-square displacement exhibits subdiffusive behavior, reminiscent of dynamics in supercooled liquids at intermediate timescales. This behavior, which can be rationalized by noting that the core of a growing collection of cells is likely to be in a jammed state, is expected on timescales less than the cell division time.

A theory to capture the essence of tumor invasion must consider the interplay of the cell mechanics, adhesive interaction between cells, and the dynamics associated with cell division and apoptosis, over a wide range of timescales. In an attempt to capture collective dynamics in 
cells, a number of models based on cellular automata $[13,14]$, vertex and Voronoi models [15-19], subcellular element models [20], cell dynamics based on the Potts model $[21,22]$, and the phase field description for collective migration $[23,24]$ have been proposed. Previous works have investigated a number of two-dimensional (2D) models in various contexts $[9,16,25]$, including probing the dynamics in a homeostatic state where cell birth-death processes are balanced [26,27]. Existing three-dimensional (3D) models focus solely on tumor growth kinetics, spatial growth patterns $[28,29]$, or on cell migration at low cellular density on timescales shorter than the cell division time [30-32]. A recent interesting study [26] shows that cell dynamics in a confluent tissue is always fluidized by cell birth and death processes, on timescales comparable to cell division time. A more recent two-dimensional model [27] investigates the glass-to-liquid transition in confluent tissues using simulations. However, both of these instructive models $[26,27]$ focus on the steady-state regime, where the number of tumor cells is kept constant by balancing the birth and death rates. Consequently, they do not address the nonequilibrium dynamics of the evolving tumor in the early stages, which is of great interest in cancer biology [33-35].

Here, we use a minimal physical 3D model that combines cell mechanical characteristics and cell-cell adhesive interactions and variations in cell birth rates to probe the nonsteady-state tumor evolution. Such a model, which has the distinct advantage that it can be generalized to include mutational effects naturally, was first introduced by Drasdo and Höhme [28]. Two of our primary goals are to understand quantitatively the complex invasion dynamics of the tumor into a collagen matrix and to provide a mechanism for the observation of superdiffusive behavior. We use free boundary conditions for tumor evolution to study dynamical fingerprints of invasion in order to quantitatively compare the results to experimental observations. We model the proliferation behavior of tumor cells and investigate the effect of pressure-dependent growth inhibition. Good agreement between our results and in vitro experiments on threedimensional growth of multicellular tumor spheroids lends credence to the model. On timescales less than the cell division time, the dynamics of cell movement within the tumor exhibits glassy behavior, reflected in a subdiffusive behavior of the mean-square displacement $\Delta(t)$. However, at times exceeding the cell division time, we find superdiffusive behavior with $\Delta(t) \sim t^{\alpha}(\alpha=1.26 \pm 0.05)$. The duration for which subdiffusion persists decreases as the cell growth rate increases, in sharp contrast to the dynamics in confluent tissues. Detailed analyses of the individual cell trajectories reveal complex heterogeneous spatial and time-dependent cell migration patterns, thus providing insights into how cells are poised for invasion into regions surrounding the tumor. We find that activity due to cell division coupled with cell mechanical interactions plays a critical role in the nonequilibrium dynamics and the physical structure of the polarized tumor invasion process. The dynamical properties of cells in our model share considerable similarities to those found in nonliving soft materials such as soap foams and toothpaste [36]. In all these cases, the transition from a glasslike behavior to superdiffusion occurs as a result of cell growth and death (or the creation and destruction of particles), resulting in nonconservation of number density without the possibility of reaching homeostasis. In other words, the nonequilibrium dynamics arising due to forces that result from key biological events (cell birth and death) that we have investigated here are qualitatively different from the dynamics in systems that do not take into account such forces.

\section{MULTICELLULAR TUMOR GROWTH MODEL}

We simulated the spatiotemporal dynamics of a multicellular tumor using a three-dimensional (3D) agent-based model, in which the cells in the tumor are represented as interacting objects. In this model, the cells grow stochastically as a function of time and divide upon reaching a critical size. The cell-to-cell interaction is characterized by direct elastic and adhesive forces. We also consider cell-tocell and cell-to-matrix damping as a way of accounting for the effects of friction experienced by a moving cell because of other cells and by the extracellular matrix (ECM) (or collagen matrix), respectively.

Each cell is modeled as a deformable sphere with a timedependent radius. Several physical properties, such as the radius, elastic modulus, membrane receptor and ligand concentration, and adhesive interaction, characterize each cell. Following previous studies [28,29,37], we use the Hertzian contact mechanics to model the magnitude of the elastic force between two spheres of radii $R_{i}$ and $R_{j}$ [Fig. 1(a)],

$$
F_{i j}^{\mathrm{el}}=\frac{h_{i j}^{3 / 2}(t)}{\frac{3}{4}\left(\frac{1-\nu_{i}^{2}}{E_{i}}+\frac{1-\nu_{j}^{2}}{E_{j}}\right) \sqrt{\frac{1}{R_{i}(t)}+\frac{1}{R_{j}(t)}}},
$$

where $E_{i}$ and $\nu_{i}$, respectively, are the elastic modulus and Poisson ratio of cell $i$. The overlap between the spheres, if they interpenetrate without deformation, is $h_{i j}$, which is defined as $\max \left[0, R_{i}+R_{j}-\left|\vec{r}_{i}-\vec{r}_{j}\right|\right]$, with $\left|\vec{r}_{i}-\vec{r}_{j}\right|$ being the center-to-center distance between the two spheres [see Fig. 1(a)]. The repulsive force in Eq. (1) is valid for small virtual overlaps such that $h_{i j} \ll \min \left[R_{i}, R_{j}\right]$ and is likely to underestimate the actual repulsion between the cells [29]. Nevertheless, the model incorporates measurable mechanical properties of the cell, such as $E_{i}$ and $\nu_{i}$, and, hence, we use this form for the repulsive force.

Cell adhesion, mediated by receptors on the cell membrane, is the process by which cells interact and attach to one another. For simplicity, we assume that the receptor and ligand molecules are evenly distributed on the cell surface. Consequently, the magnitude of the adhesive force $F_{i j}^{\mathrm{ad}}$ between two cells $i$ and $j$ is expected to scale as a function of their contact area $A_{i j}$ [38]. We estimate $F_{i j}^{\text {ad }}$ using [29] 
(a)

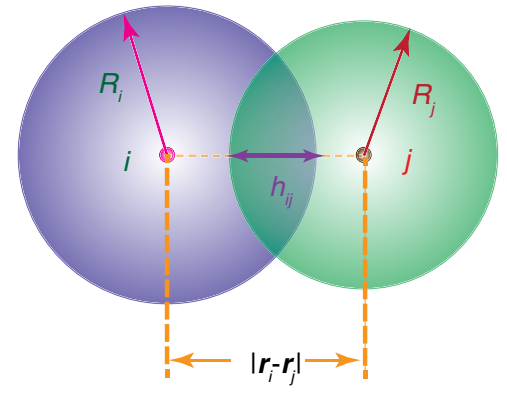

(b)

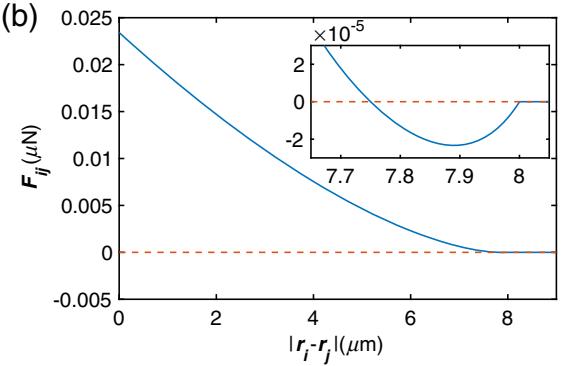

FIG. 1. (a) Two interpenetrating cells $i$ and $j$ with radii $R_{i}$ and $R_{j}$, respectively. The distance between the centers of the two cells is $\left|\mathbf{r}_{i}-\mathbf{r}_{j}\right|$, and their overlap is $h_{i j}$. (b) Force on cell $i$ due to $j, \mathbf{F}_{i j}$, for $R_{i}=R_{j}=4 \mu \mathrm{m}$ using mean values of the elastic modulus, Poisson ratio, and receptor and ligand concentration (see Table I). $\mathbf{F}_{i j}$ is plotted as a function of the distance between the centers of the two cells. The inset shows the region where $\mathbf{F}_{i j}$ is attractive. When $\left|\mathbf{r}_{i}-\mathbf{r}_{j}\right| \geq R_{i}+R_{j}=8 \mu \mathrm{m}$, the cells are no longer in contact and, hence, $\mathbf{F}_{i j}=0$.

$$
F_{i j}^{\mathrm{ad}}=A_{i j} f^{\mathrm{ad}} \frac{1}{2}\left(c_{i}^{\mathrm{rec}} c_{j}^{\mathrm{lig}}+c_{j}^{\mathrm{rec}} c_{i}^{\mathrm{lig}}\right),
$$

where $c_{i}^{\text {rec }}\left(c_{i}^{\text {lig }}\right)$ is the receptor (ligand) concentration (assumed to be normalized with respect to the maximum receptor or ligand concentration so that $0 \leq c_{i}^{\text {rec }}, c_{i}^{\text {lig }} \leq 1$ ). The coupling constant $f^{\text {ad }}$ allows us to rescale the adhesion force to account for the variabilities in the maximum densities of the receptor and ligand concentrations. We calculate the contact surface area $A_{i j}$ using the Hertz model prediction, $A_{i j}=\pi h_{i j} R_{i} R_{j} /\left(R_{i}+R_{j}\right)$. The Hertz contact surface area is smaller than the proper spherical contact surface area. However, in dense tumors, many spheres overlap and, thus, the underestimation of the cell surface overlap may be advantageous in order to obtain a realistic value of the adhesion forces [29].

Repulsive and adhesive forces considered in Eqs. (1) and (2) act along the unit vector $\vec{n}_{i j}$ pointing from the center of cell $j$ to the center of cell $i$ [Fig. 1(a)]. The force exerted by cell $j$ on cell $i, \mathbf{F}_{i j}$, is shown in Fig. 1(b). The total force on the $i$ th cell is given by the sum over its nearest neighbors $[N N(i)]$,

$$
\vec{F}_{i}=\Sigma_{j \epsilon N N(i)}\left(F_{i j}^{\mathrm{el}}-F_{i j}^{\mathrm{ad}}\right) \vec{n}_{i j} .
$$

We developed a distance sorting algorithm to efficiently provide a list of nearest neighbors in contact with the $i$ th cell for use in the simulations. For any given cell $i$, an array containing the distances from cell $i$ to all the other cells is initially created. We then calculated $R_{i}+R_{j}-\left|\vec{r}_{i}-\vec{r}_{j}\right|$ and sorted the cells $j$ satisfying $R_{i}+R_{j}-\left|\vec{r}_{i}-\vec{r}_{j}\right|>0$, a necessary condition for any cell $j$ to be in contact with cell $i$.

\section{SIMULATIONS}

\section{A. Equations of motion}

The spatial dynamics of the cell is computed based on the equation of motion $[29,39,40]$ for a cell of mass $m_{i}$,

$m_{i} \ddot{r}_{i}^{\alpha^{\prime}}=F_{i}^{\alpha^{\prime}}(t)-\Sigma_{\beta^{\prime}} \gamma_{i}^{\alpha^{\prime} \beta^{\prime}} \dot{r}_{i}^{\beta^{\prime}}(t)-\Sigma_{\beta^{\prime}} \Sigma_{j} \gamma_{i j}^{\alpha^{\prime} \beta^{\prime}}\left[\dot{r}_{i}^{\beta^{\prime}}(t)-\dot{r}_{j}^{\beta^{\prime}}(t)\right]$,

where the greek indices $\left[\alpha^{\prime}, \beta^{\prime}\right]=[x, y, z]$ are for coordinates, and the latin indices $[i, j]=[1,2, \ldots, N]$ are the cell indices. In Eq. (4), $\gamma_{i}^{\alpha^{\prime} \beta^{\prime}}$ is the cell-to-medium friction coefficient, and $\gamma_{i j}^{\alpha^{\prime} \beta^{\prime}}$ is the cell-to-cell friction coefficient. The adhesive and repulsive forces are included in the term $F_{i}^{\alpha^{\prime}}$. The cell-to-ECM friction coefficient is assumed to be given by the Stokes relation,

$$
\gamma_{i}^{\alpha^{\prime} \beta^{\prime}, \text { visc }}=6 \pi \eta R_{i} \delta^{\alpha^{\prime} \beta^{\prime}}
$$

based on the friction of a sphere in a medium of viscosity $\eta$. Here, $\delta^{\alpha^{\prime} \beta^{\prime}}$ is the Kronecker delta.

Because the Reynolds number for cells in a tissue is small [39], overdamped approximation is appropriate, implying that the neglect of the inertial term $m_{i} \ddot{r}_{i}^{\alpha^{\prime}} \approx 0$ is justified [29] (see Appendix A for further discussion). Since additional adhesive forces are also present, cell movement is further damped [41]. We simplify the equations of motion [Eq. (4)] by replacing the intercellular drag term with a modified friction term, given that the movement of the bound cells is restricted. The modified friction term will contribute to the diagonal part of the damping matrix with $\gamma_{i}^{\alpha^{\prime} \beta^{\prime}}=\gamma_{i}^{\alpha^{\prime} \beta^{\prime}, \text { visc }}+\gamma_{i}^{\alpha^{\prime} \beta^{\prime} \text {,ad }}$, where

$$
\begin{aligned}
\gamma_{i}^{\alpha^{\prime} \beta^{\prime}, a d}= & \gamma^{\max } \Sigma_{j \in N N(i)}\left(A_{i j} \frac{1}{2}\left(1+\frac{\vec{F}_{i} \cdot \vec{n}_{i j}}{\left|\vec{F}_{i}\right|}\right)\right. \\
& \left.\times \frac{1}{2}\left(c_{i}^{\mathrm{rec}} c_{j}^{\operatorname{lig}}+c_{j}^{\mathrm{rec}} c_{i}^{\mathrm{lig}}\right)\right) \delta^{\alpha^{\prime} \beta^{\prime}} .
\end{aligned}
$$

Notice that the added friction coefficient $\gamma_{i}^{\alpha^{\prime} \beta^{\prime}, a d}$ is proportional to the cell-to-cell contact surface, implying that a cell in contact with multiple cells would move less. The nonisotropic nature of the adhesive friction is evident from the factor $\left\{1+\left[\left(\vec{F}_{i} \cdot \vec{n}_{i j}\right) /\left(\left|\vec{F}_{i}\right|\right)\right]\right\}$, where the maximum 
contribution occurs when the net force $\vec{F}_{i}$ is parallel to a given unit vector $\vec{n}_{i j}$ among the nearest neighbors. With these approximations, the equations of motion [Eq. (4)] are now diagonal,

$$
\dot{\vec{r}}_{i}=\frac{\vec{F}_{i}}{\gamma_{i}}
$$

\section{B. Cell cycle}

In our model, cells can be either in the dormant $(D)$ or in the growth $(G)$ phase. We track the sum of the normal pressure that a particular cell $i$ experiences due to contact with its neighbors, using

$$
p_{i}=\Sigma_{j \epsilon N N(i)} \frac{\left|\vec{F}_{i j} \cdot \vec{n}_{i j}\right|}{A_{i j}} .
$$

If the local pressure $p_{i}$ exceeds a critical limit $\left(p_{c}\right)$, the cell stops growing and enters the dormant phase (see the left panel in Fig. 2). For growing cells, their volume increases at a constant rate $r_{V}$. The cell radius is updated from a Gaussian distribution with the mean rate $\dot{R}=\left(4 \pi R^{2}\right)^{-1} r_{V}$. Over the cell cycle time $\tau$,

$$
r_{V}=\frac{2 \pi\left(R_{m}\right)^{3}}{3 \tau}
$$

where $R_{m}$ is the mitotic radius. A cell divides once it grows to the fixed mitotic radius. To ensure volume conservation, upon cell division, we use $R_{d}=R_{m} 2^{-1 / 3}$ as the radius of the daughter cells (see the right panel in Fig. 2). The two resulting cells are placed at a center-to-center distance $d=2 R_{m}\left(1-2^{-1 / 3}\right)$. The direction of the new cell location is chosen randomly from a uniform distribution on the unit sphere. One source of stochasticity in the cell movement in

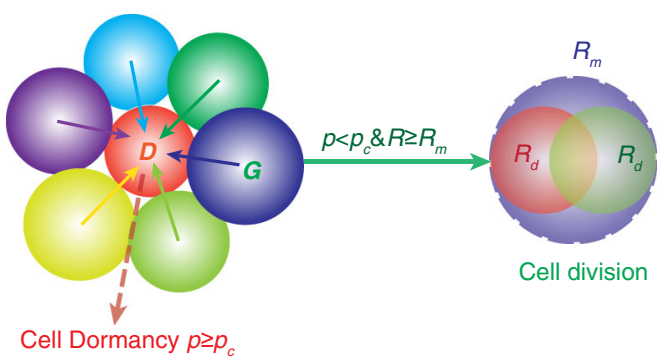

FIG. 2. Cell dormancy (left panel) and cell division (right panel). If the local pressure $p_{i}$ that the $i$ th cell experiences (due to contacts with the neighboring cells) exceeds the critical pressure $p_{c}$, it enters the dormant state $(D)$. Otherwise, the cells grow $(G)$ until they reach the mitotic radius $R_{m}$. At that stage, the mother cell divides into two identical daughter cells with the same radius $R_{d}$. We assume that the total volume upon cell division is conserved. A cell that is dormant at a given time can transit from that state at subsequent times. our model is due to random choice for the mitotic direction. Together with stochasticity in the cell cycle duration, we obtain fairly isotropic tumor spheroids.

\section{Tumor invasion distance}

The invasion or spreading distance of the growing tumor $\Delta r(t)$ is determined by measuring the average distance from the tumor center of mass $\left[\vec{R}_{C M}=(1 / N) \Sigma_{i} \vec{r}_{i}\right]$ to the cells at the tumor periphery,

$$
\Delta r(t)=\frac{1}{N_{b}} \sum_{i}^{N_{b}}\left|\vec{r}_{i}-\vec{R}_{C M}\right|,
$$

where the sum $i$ is over $N_{b}$, the number of cells at the tumor periphery. In order to find the cells at the tumor periphery $\left(N_{b}\right)$, we denote the collection of all cells $N$ as a set of vertices $\{1,2,3 \ldots \ldots \ldots N\}$ in $\mathbb{R}^{3}$, where the vertices represent the center point of each cell. We generate a 3D structure of tetrahedrons using these vertices, where each tetrahedron is comprised of four vertices. Let $T$ be the total number of tetrahedrons. Since each tetrahedron has four faces, the total number of faces is $4 T$. Any face that is not on the boundary of the 3D structure is shared by two tetrahedrons, but the boundary faces are not shared. Thus, our aim is to find the set of unshared boundary faces out of the total number of faces $4 T$. Once the boundary faces are obtained, we know the list of vertices and, hence, the positions of cells at the tumor periphery, allowing us to compute $\Delta r(t)$ in Eq. (10).

\section{RESULTS}

\section{A. Calibration of the model parameters}

We compare the normalized volume of the growing tumor to experimental data [42], as a way of assessing if the parameters (Table I) used in our model are reasonable. The tumor volume $V(t)$, normalized by the initial volume

TABLE I. The parameters used in the simulation.

\begin{tabular}{lcc}
\hline \hline Parameters & Values & References \\
\hline Time step $(\Delta t)$ & $1-10 \mathrm{~s}$ & This paper \\
Critical radius for division $\left(R_{m}\right)$ & $5 \mu \mathrm{m}$ & {$[29]$} \\
Extracellular matrix $(\mathrm{ECM})$ & $0.005 \mathrm{~kg} /(\mu \mathrm{m} \mathrm{s})$ & {$[44]$} \\
$\quad$ viscosity $(\eta)$ & & \\
Benchmark cell cycle time $\left(\tau_{\min }\right)$ & $54,000 \mathrm{~s}$ & {$[45-47]$} \\
Adhesive coefficient $\left(f^{\text {ad }}\right)$ & $10^{-4} \mu \mathrm{N} / \mu \mathrm{m}^{2}$ & {$[29]$} \\
Mean cell elastic modulus $\left(E_{i}\right)$ & $10^{-3} \mathrm{MPa}$ & {$[44]$} \\
Mean cell Poisson ratio $\left(\nu_{i}\right)$ & 0.5 & {$[29]$} \\
Death rate $(b)$ & $10^{-6} \mathrm{~s}^{-1}$ & This paper \\
Mean receptor concentration $\left(c^{\mathrm{rec}}\right)$ & $1.0(\mathrm{normalized})$ & {$[29]$} \\
Mean ligand concentration $\left(c^{\text {lig }}\right)$ & $1.0(\mathrm{normalized})$ & {$[29]$} \\
Adhesive friction $\gamma^{\text {max }}$ & $10^{-4} \mathrm{~kg} /\left(\mu \mathrm{m}^{2} \mathrm{~s}\right)$ & This paper \\
Threshold pressure $\left(p_{c}\right)$ & $10^{-4} \mathrm{MPa}$ & {$[29,42]$} \\
\hline \hline
\end{tabular}


of the spheroid $\left(V_{0}\right)$, was tracked experimentally using colon carcinoma cells [42] through experimental methods that are very different from the way we simulated tumor growth. The tumor growth was measured by imposing stress [42], known to inhibit cancer growth [43]. These effects are included in our model, which allows us to make quantitative comparisons between our simulations and experiments. The tumor volume is obtained in the simulation from $R_{g}$, the radius of gyration,

$$
R_{g}^{2}=\frac{1}{N} \sum_{i}^{N}\left(\vec{r}_{i}-\vec{R}_{C M}\right)^{2}
$$

where $N$ is the total number of cells. The volume $V(t)$ is given by $(4 \pi / 3) R_{g}^{3}(t)$. Our simulation of the growth of the spheroid tumor volume in the early stages is in good agreement with experimental data [see Fig. 3(a)]. Thus, our model captures quantitative aspects of tumor growth.

In the experiment [42], $V(t) / V_{0}$ was measured for external pressure ranging from 0 to $20 \mathrm{kPa}$. In Fig. 3(a), we compared our simulation results with the 500-Pa result from experiments [42]. This is rationalized as follows.
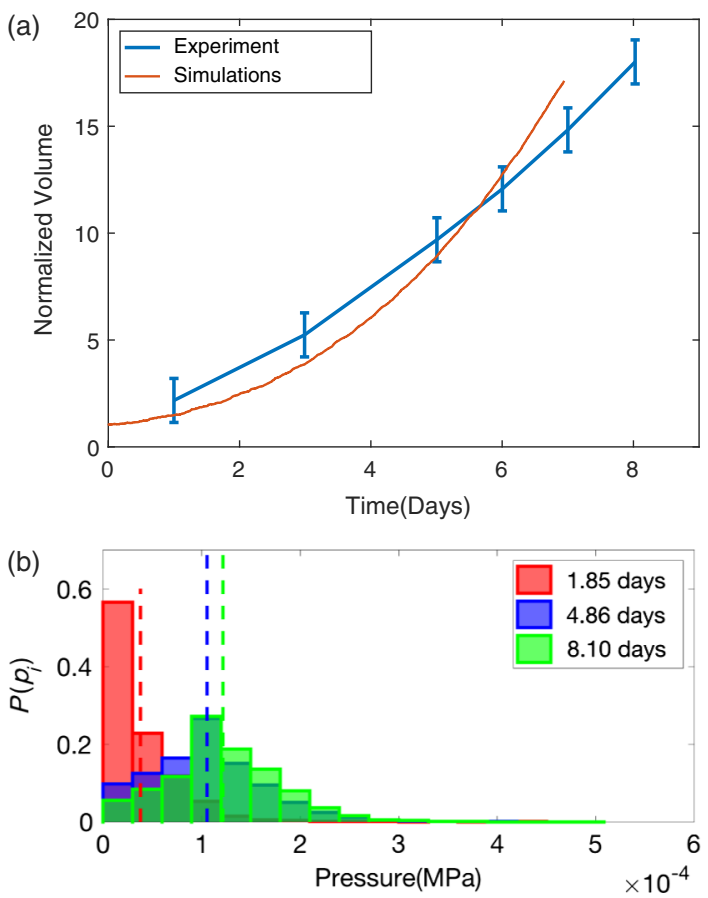

FIG. 3. (a) Normalized volume $V(t) / V_{0}$ of a tumor spheroid as a function of time. The result of the simulation (red) agrees nearly quantitatively with experimental data obtained for the tumor spheroid growth at an applied pressure of $500 \mathrm{~Pa}$ [42]. We used a critical pressure $p_{c}=100 \mathrm{~Pa}$ and cell division time of $\tau=\tau_{\min }$ in these simulations. The reason for comparing the results from the $p_{c}=100 \mathrm{~Pa}$ simulations with the growth dynamics obtained in the colon carcinoma cells with an external pressure of $500 \mathrm{~Pa}$ is explained in the text. (b) Distribution of pressure as a function of total growth time with cell division time $\tau=\tau_{\min }=0.625$ days. The mean values are indicated by the dashed lines.
Unlike in experiments, the pressure is internally generated as the tumor grows [Fig. 3(b)] with a distribution that changes with time. The mean value of the pressure [see the dashed lines in Fig. 3(b)] at the longest time is approximately $100 \mathrm{~Pa}$. Thus, it is most appropriate to compare our results obtained using $p_{c}=100 \mathrm{~Pa}$ with experiments in which the external pressure is set to $500 \mathrm{~Pa}$.

\section{B. Predicted pressure-dependent growth dynamics is consistent with experiments}

A visual representation of the tumor growth process generated in simulations is vividly illustrated in the Videos (see Videos 1 and 2) in Appendix B. Snapshots of the evolving collection of cells at different times are presented in Figs. 4(a)-(d). As the tumor evolves, the cells aggregate into a spheroidal shape because the cell division plane is isotropically distributed [Fig. 4(d) and the Video 1 in Appendix B]. In spheroidal cell aggregates, it is known that pressure inhibits cell proliferation $[42,48,49]$. We expect the pressure [see Eq. (8)] experienced by the cells in the interior of the tumor to be elevated because of crowding effects, causing the cells to enter a dormant state if the pressure from the neighbors reaches a preset threshold value $p_{c}$. Tumor growth behavior is strongly dependent on the value of $p_{c}$ [see Fig. 4(e)]. At $p_{c}=10^{-3} \mathrm{MPa}$, the total number $[N(t)]$ of tumor cells during growth is well approximated as an exponential $N(t) \propto \exp ($ const $\times t)$. As $p_{c}$ is lowered, implying growth is inhibited at smaller pressures, an increase in the tumor size is described by a power law, $N(t) \propto t^{\beta}$, at long timescales, while $N(t)$ retains exponential growth at early stages [see the inset of Fig. 4(e)]. Our simulations also show that $\beta$ is $p_{c}$ dependent, increasing as $p_{c}$ increases. Power-law growth in the 3D tumor spheroid size has been observed in many tumor cell lines, with $\beta$ varying from 1 to 3 [50-54]. The overall growth of the tumor slows down as the value of the pressure experienced by cells increases, which is also consistent with recent experimental results [49]. The known results in Fig. 4(e) are in near quantitative agreement with several experiments, thus validating the model.

\section{Cell motility within the tumor spheroid}

Using direct imaging techniques, it has become possible to monitor the overall invasion of the tumor, as well as the movement of individual cells within the spheroid $[55,56]$. In order to compare our results to experiments, we calculated the mean-square displacement, $\Delta(t)=\left\langle[\mathbf{r}(t)-\mathbf{r}(0)]^{2}\right\rangle$, of individual cells. By tracking the movement of all the initial cells within the tumor, we calculated $\Delta(t)$ by averaging over hundreds of trajectories. The growth-rate-dependent $\Delta(t)$ [displayed in Fig. 5(a) on a log-log scale] shows that there is a rapid increase in $\Delta(t)$ at early times $\left(t \leq 0.01 \tau_{\min }\right.$, where $\tau_{\min }$ is the benchmark value of the cell cycle time given in Table I) because the cells move unencumbered, driven by repulsive 
(a)

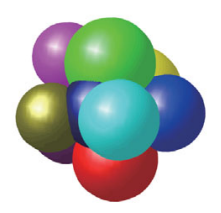

(c)

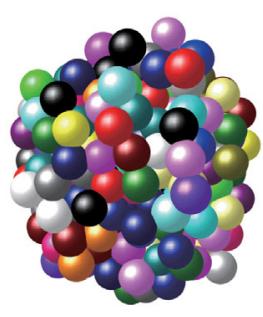

(b)

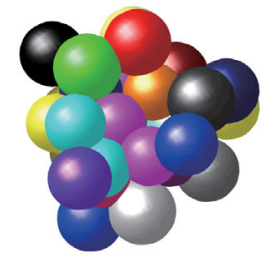

(d)

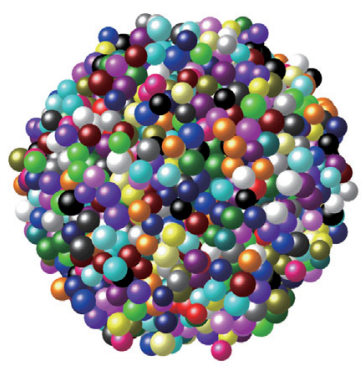

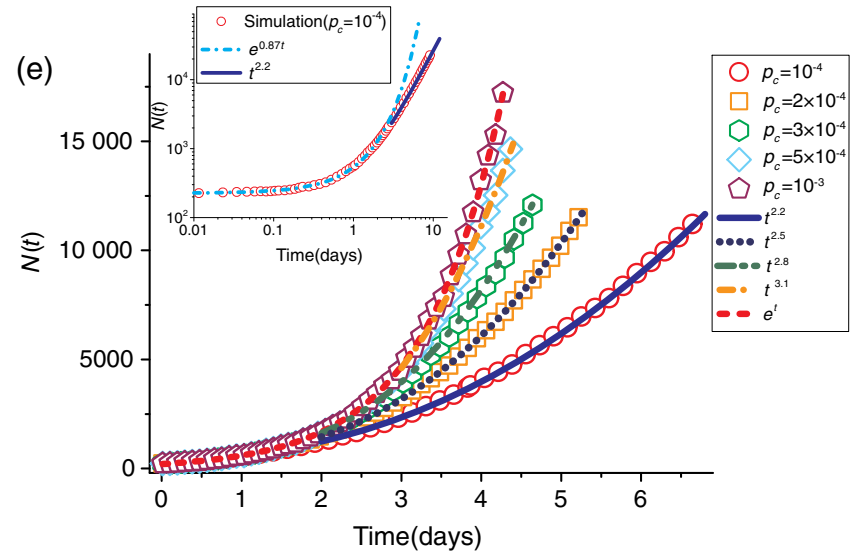

FIG. 4. Dynamics of tumor growth. (a)-(d): Instantaneous snapshots of the tumor during growth at different times. Each cell is represented by a sphere. There are approximately 2000 cells in (d). The color of each cell is to aid visualization. In (a)-(d), the cell sizes are rescaled for illustration purposes only. Note that, even at $t=0$ (a), the sizes of the cells are different because they are drawn from a Gaussian distribution. (e): The total number of tumor cells $N(t)$ as a function of time at different values of the threshold pressure $p_{c}$, which increases from bottom to top $\left(10^{-4}, 2 \times 10^{-4}, 3 \times 10^{-4}, 5 \times 10^{-4}, 10^{-3} \mathrm{MPa}\right)$. The dashed red line is an exponential function, while the other lines show power-law behavior $N(t) \approx t^{\beta}$, where $\beta$ ranges from 2.2 to $2.5,2.8$, and 3.1 (from bottom to top). The inset in (e) shows $N(t)$ with $p_{c}=10^{-4}$ on a log-log scale with both exponential and power-law fits. The dashed-dotted curve in the inset is an exponential function, while the power-law trend is illustrated by the solid line. The average cell cycle time $\tau=\tau_{\min }=54000 \mathrm{~s}$, and the other parameter values are taken from Table I.

interaction with other cells. At intermediate timescales $\left(0.01 \tau_{\min }<t<\tau\right.$, with $\tau$ being the average cell cycle time), $\Delta(t)$ exhibits subdiffusive behavior $\left[\Delta(t) \sim t^{s}\right.$, with $\left.s<1\right]$. The signatures of the plateaus in $\Delta(t)$ (together with other characteristics discussed later) in this time regime indicate that cells are caged by the neighbors [see the left inset in Fig. 5(a)] and consequently undergo only small

displacements. Such a behavior is reminiscent of a supercooled liquid undergoing a glass transition, as illustrated in colloidal particles using direct imaging as their densities approach the glass transition [57,58]. As $\tau$ increases, the plateau persists for longer times because of a decrease in the outward stress, which slows the growth of the tumor. When $t$ exceeds $\tau$, the average cell doubling time, the $\Delta(t)$ exhibits superdiffusive motion, $\Delta(t) \approx t^{\alpha}(\alpha>1)$. In order to determine $\alpha$, we performed multiple simulations and calculated $\Delta(t)$ for each of them by generating a large number of trajectories. All the independent simulations show that $\Delta(t)$ has the characteristic plateau at intermediate times followed by superdiffusion at long times in Fig. 5(b). From each of these simulations, we determine $\alpha$, whose distribution is shown in Fig. 5(c). In all cases, we find that $\alpha$ is greater than unity. The estimate from the distribution in Fig. 5(c) is $\alpha=1.26 \pm 0.05$, where 0.05 is the standard deviation.

On long timescales, cells can escape the cage created by their neighbors, as illustrated in the middle inset of Fig. 5(a). Our observation of superdiffusion in $\Delta(t)$ at long times agrees well with the experimental result $(\alpha \approx 1.40 \pm 0.04)$ obtained for fibrosarcoma cells in a growing tumor spheroid [33]. The onset of superdiffusive behavior in $\Delta(t)$ shifts to earlier times as we decrease the average cell cycle time [see Fig. 5(a)], implying that cell division is the mechanism resulting in superdiffusion (see below for further discussion of this crucial finding).

We provide another rationale for the robustness of the long time superdiffusive behavior. This comes from examining the time-dependent changes in the invasion distance, $\Delta r(t)$ in Eq. (10). The finding that the invasion distance does not increase as a function of time with exponent $\Delta r \propto$ $t^{0.5}$ but rather at a higher exponent at the long time regime necessarily implies that cells do not execute random walk motion (see Ref. [33]). The dependence of $\Delta r(t)$ on $t$ in Fig. 6 shows that, for $t \leq \tau_{\min }$, the invasion radius is roughly constant. As cells divide, the tumor invasion distance $\Delta r(t)$ increases as $t^{\xi}$ with $\xi \sim 0.63$ (which implies that $\alpha \approx 1.26$ ) for $t>\tau_{\min }$, a value that is not inconsistent with experiments [33].

\section{Theoretical predictions}

In order to understand the role of cell growth and apoptosis in the observed sluggish dynamics at intermediate times and superdiffusive behavior at long times, we developed a theory to study the dynamics of a colony of cells in a dissipative environment (Appendix C). The interactions between cells contain both attractive (adhesive) and excluded volume terms. Starting from the Langevin equation describing the dynamics of the $i$ th cell, and incorporating the birth reaction, $X \stackrel{k_{a}}{\rightarrow} X+X$, with the rate constant $k_{a}(=1 / \tau)$ for each cell, and the apoptosis reaction $X+X \stackrel{k_{b}}{\rightarrow} X$, with the rate $k_{b}$, an equation for the time dependence of the density $\rho(\mathbf{k}, t)$ [Eq. (C3) in Appendix C] 


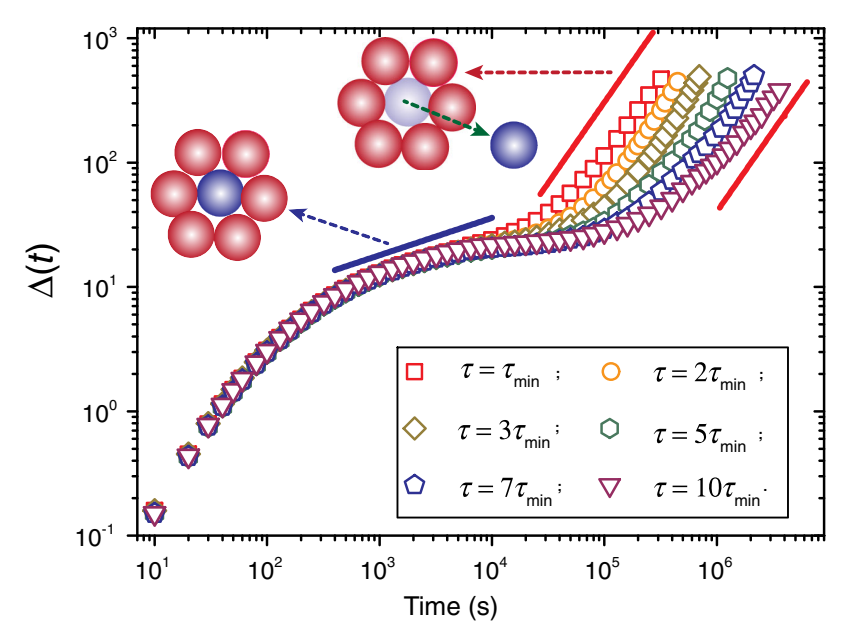

(b)

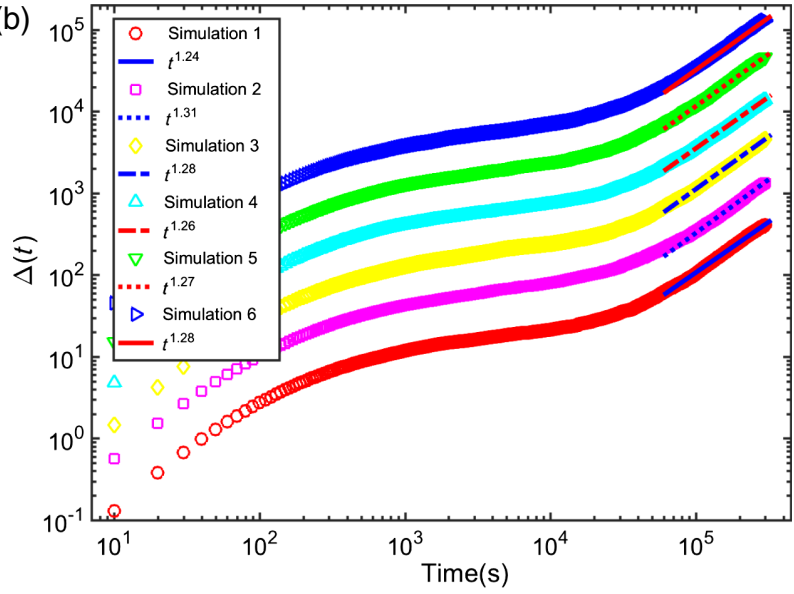

(c)

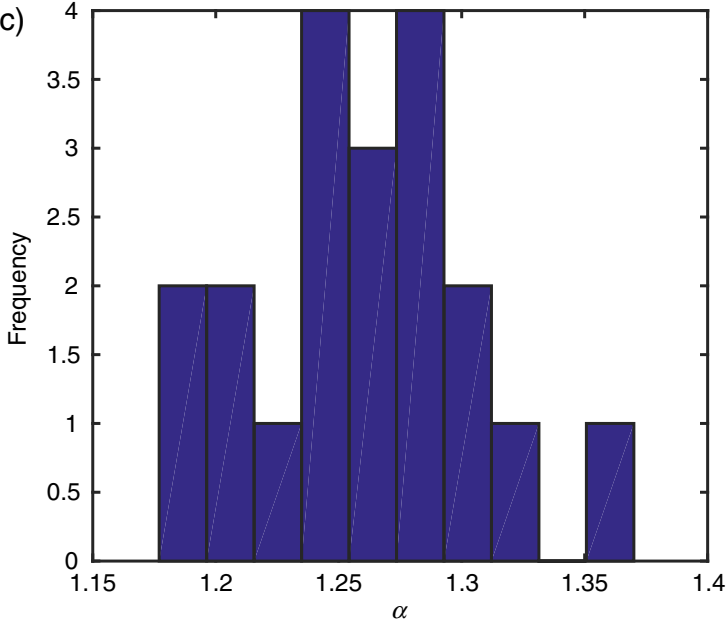

FIG. 5. Superdiffusive behavior in $\Delta(t)$ at long times. (a) The mean-square displacement [ $\Delta(t)]$ of cells. From top to bottom, the curves correspond to increasing average cell cycle time ( $\tau$ is varied from $\tau_{\min }$ to $10 \tau_{\min }$, where $\tau_{\min }=54000 \mathrm{~s}$ ). Time taken for reaching the superdiffusive regime increases by increasing $\tau$. The blue and red lines have slopes of 0.33 and 1.3, respectively. The subdiffusive (superdiffusive) behavior corresponds to dynamics in the intermediate (long) times. The left and middle inset illustrate the "cage effect" and "cage-jump" motion, respectively. The unit for the $y$-axis is $\mu \mathrm{m}^{2}$. (b) Fit of the mean-square displacement $\Delta(t)$ to $t^{\alpha}$ for several simulation runs. Each $\Delta(t)$ plot is averaged over approximately 300 cell trajectories. The six $\Delta(t)$ plots consistently show $\alpha>1.20$ over approximately 1800 cell trajectories. The plots are separated for clarity. (c) Distribution of $\alpha$ values from multiple independent simulations. The mean value of $\alpha$ is 1.26 , with a standard deviation of \pm 0.05 .

can be derived. The cell division and apoptosis processes drive the system far from equilibrium, thus violating the fluctuation dissipation theorem (FDT). As a consequence, we cannot use the standard methods used to calculate the response and correlation functions from which the $t$ dependence of $\Delta(t)$ can be deduced. To overcome this difficulty, we used the Parisi-Wu stochastic quantization method [59], in which the evolution of $\rho(\mathbf{k}, \omega)(\omega$ is the frequency) is described in a fictitious time in which FDT is preserved. From the analysis of the resulting equation (Appendix $\mathrm{C}$ contains the sketch of the calculations), the scaling of $\Delta(t)$ may be obtained as

$$
\Delta(t)=\left\langle[\mathbf{r}(t)-\mathbf{r}(0)]^{2}\right\rangle \sim t^{2 / z} .
$$

In the intermediate time regime, $z=5 / 2$, implying that $\Delta(t) \approx t^{4 / 5}$. The predicted subdiffusive behavior of $\Delta(t)$ is qualitatively consistent with simulation results. It is likely that the differences in the scaling exponent between simulations $(2 / z \approx 0.33)$ and theoretical predictions $(2 / z \approx 0.80)$ in this nonuniversal time regime may be due to the differences in the cell-to-cell interactions used in the two models.

In the long time limit, the cell birth-death process [the fourth term in Eq. (C3)] dominates the interactions between cells. As a result, we expect that the exponent $2 / z$ should be universal, independent of the forces governing cell motility. Our theory predicts that $z=3 / 2$, which shows that $\Delta(t) \approx t^{4 / 3}$, in excellent agreement with the simulations [Fig. 5(a)] and experiments [33]. It is clear from our theory 


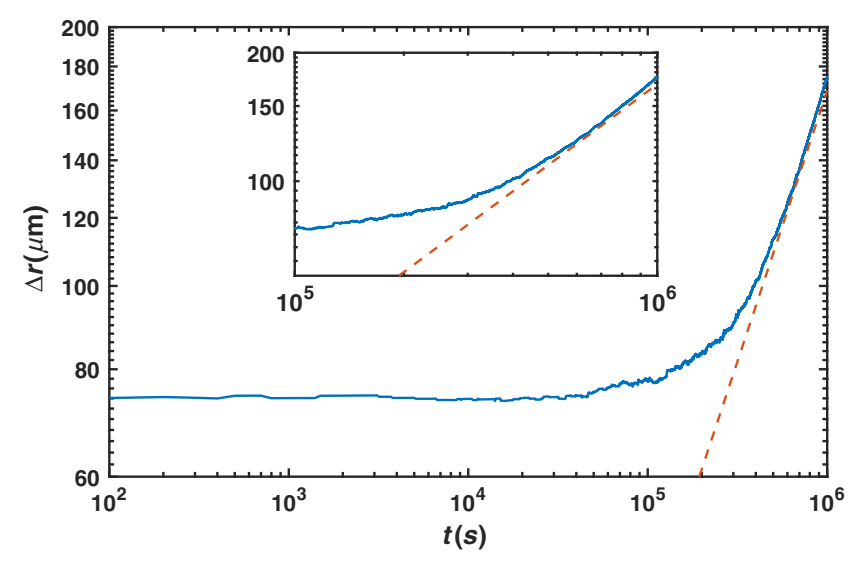

FIG. 6. The invasion distance $\Delta r(t)$ [Eq. (10)] as a function of time. The exponent of the invasion distance, as indicated by the dashed line, determined using $\Delta r \propto t^{\xi}$, is $\xi \approx 0.63$. Note that this value and the one extracted from experiments [33] are in reasonable agreement. The inset shows $\Delta r(t)$ for $t>\tau_{\min }$, for average cell cycle time $\tau=1 \tau_{\min }$.

that the interaction-independent biologically important birth-death processes drive the observed fluidization during tumor (or tissue) development, resulting in superdiffusive cell motion at long times. The underlying mechanism for obtaining superdiffusive behavior is that cells must move persistently in a given direction for a long time, leading to polarized tumor growth, and ultimately resulting in an invasion driven predominantly by birth. We provide additional numerical evidence for this assertion below.

\section{E. Dependence of relaxation times on cell cycle time}

We first characterized the structural evolution as the tumor evolves. In order to assess the spatial variations in the positions of the cells as the tumor grows, we calculated the pair correlation function using

$$
g(r)=\frac{V}{4 \pi r^{2} N^{2}} \sum_{i}^{N} \sum_{j \neq i}^{N} \delta\left(r-\left|\vec{r}_{i}-\vec{r}_{j}\right|\right) .
$$

The pair correlation functions (Fig. 7), at different cell cycle times $(\tau)$, show that, at longer cell cycle times, the cells are packed more closely. There is a transition from a liquidlike to a glasslike structure as $\tau$ is increased, as indicated by the peaks in $g(r)$.

To further quantify the fluidization transition driven by cell birth-death processes, we calculated the isotropic selfintermediate scattering function $F_{s}(q, t)=\left\langle e^{i \mathbf{q} \cdot(\mathbf{r}(t)-\mathbf{r}(0))}\right\rangle$ at $|\mathbf{q}|=2 \pi / r_{0}$, where $r_{0}$ is the position of the first maximum in the pair correlation function (see Fig. 7). The average is taken over all the initial cells, which are alive during the entire simulation time and the angles of $\mathbf{q}$. We note that $F_{s}(q, t)$ exhibits a two-step relaxation process [Fig. 8(a)], characterized by two timescales. The initial

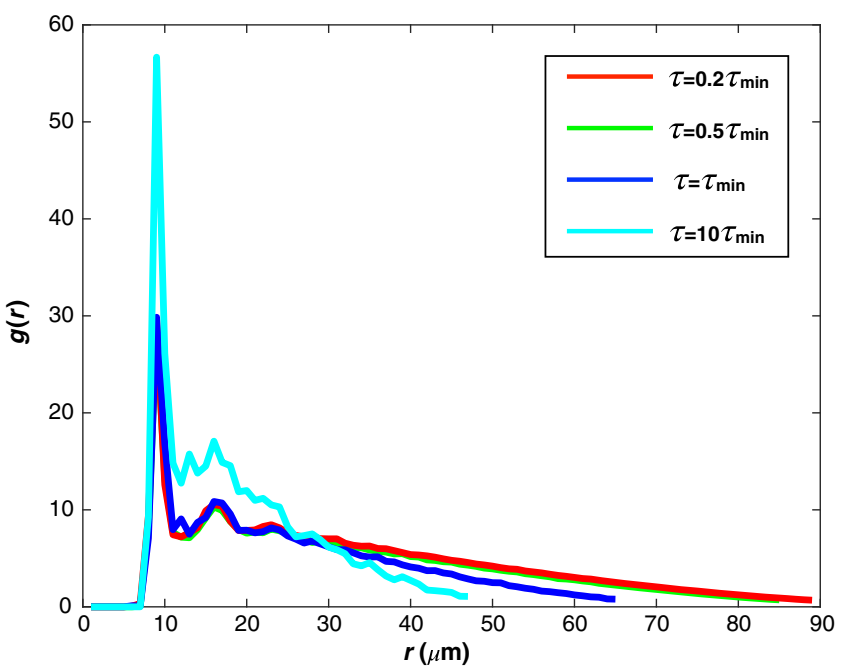

FIG. 7. Pair correlation function at four different cell division times $(\tau): 0.2 \tau_{\min }$ (red), $0.5 \tau_{\min }$ (green), $\tau_{\min }$ (blue), and $10 \tau_{\min }$ (cyan). The cells are packed more closely at longer cell cycle times as reflected by the sharper peak for the cyan line compared to the others. The distance $r$ at which $g(r)$ approaches zero is considerably smaller for $\tau=10 \tau_{\min }$. The distance at which the first peak appears is approximately $2 R_{m} \approx 10 \mu \mathrm{m}$ (Table I), which implies that, despite being soft, the cells in the interior are densely packed, as in a body centered cubic lattice.

relaxation time, corresponding to the motion of cells in a cage formed by neighboring cells, depends only weakly on the cell cycle time. The second relaxation time $\left(\tau_{\alpha}\right)$, extracted by fitting $F_{s}(q, t)$ to an exponential function $\left[F_{s}(q, t) \approx a_{0} e^{-t / \tau_{\alpha}}\right.$; see colored solid lines in Fig. 8(a)], depends strongly on the average cell cycle time. As in the relaxation of supercooled liquids, $\tau_{\alpha}$ is associated with the collective motion of cells leaving the cage [60,61]. As the average cell cycle time is reduced, $\tau_{\alpha}$ decreases [see Fig. 8(b)], and the $F_{s}(q, t)$ begins to approach a single relaxation regime, as expected for a normal fluid. The second relaxation process in $F_{s}(q, t)$ [Fig. 8(a)] can be collapsed onto one master curve by rescaling time by $\tau_{\alpha}$, resulting in the independence of $F_{s}(q, t)$ on the cell cycle time [Fig. 8(c)]. We surmise that the cage relaxation is driven by the same mechanism (the cell birth-death processes) that gives rise to the superdiffusive behavior in $\Delta(t)$.

\section{F. Diffusion of tracer cells}

Elsewhere [27], using a two-dimensional model, $\Delta(t)$ was computed for cells, as well as tracer cells. In that study, using periodic boundary conditions, the choice of birth and death rates was such that in the long time limit homeostasis was always reached where the birth and death of cells were balanced. It was found that $\Delta(t)$ for live cells (those that can be born and die) show a plateau at intermediate times, followed by normal diffusion $[\Delta(t) \sim t]$ at long times. In contrast, $\Delta_{t r}(t)$, the mean-square displacement computed 

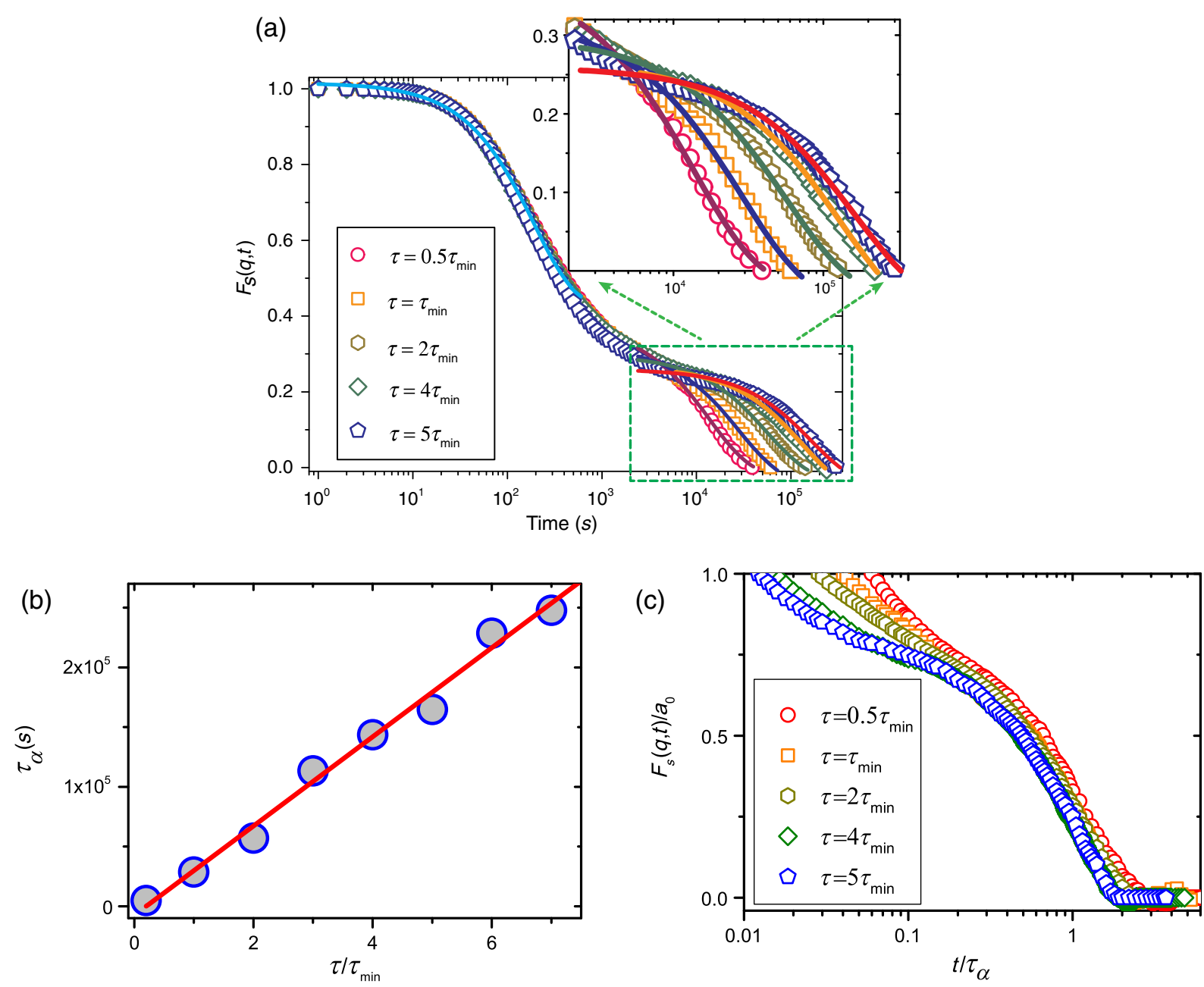

FIG. 8. Self-intermediate scattering function at different cell cycle times. (a) The self-intermediate scattering function, $F_{s}(q, t)$, shows that relaxation occurs in two steps. From left to right, the second relaxation for $F_{s}(q, t)$ slows down as $\tau$ increases (from $0.5 \tau_{\min }$ to $5 \tau_{\min }$ ). The solid lines are exponential fits. The upper inset shows a zoom-in of the dashed-line rectangle at long timescales. (b) The second relaxation time $\tau_{\alpha}$ obtained from (a) as a function of cell division time (rescaled by $\tau_{\min }$ ). The red solid line is a linear fit $\left(\tau_{\alpha} \propto 0.69 \tau\right)$. (c) The rescaled self-intermediate scattering function $F_{s}(q, t) / a_{0}$ as a function of the rescaled time $\mathrm{t} / \tau_{\alpha}$.

for tracer cells (ones that have all the characteristics of cells, except that they are alive throughout the simulations and do not grow or divide) shows no caging effects but grows linearly with time [27], suggestive of normal diffusion. In light of this dramatically different behavior reported in [27], we performed simulations using our model by including 100 randomly placed tracer cells. The interactions between the tracer cells with each other and the cells that undergo birth and death are identical. The calculated dependence of $\Delta_{t r}(t)$ for tracer cells, as a function of $t$ [Fig. 9(a)], is qualitatively similar to that for cells [compare Figs. 5(a) and 9(a)]. In particular, at varying values of $\tau, \Delta_{t r}(t)$ exhibits a plateau, followed by superdiffusive behavior, $\Delta_{t r}(t) \sim t^{\alpha_{t r}}$, at long times. However, we find that $\alpha_{t r}(>1.4)$ depends on $\tau$, in contrast to the universal exponent for cell dynamics. Similarly, $F_{s}(q, t)$ for tracer cells also displays two-step relaxation for the three values of $\tau$ investigated, as shown in Fig. 9(b). Interestingly, the values of the first relaxation times are longer than for the corresponding dynamics associated with the cells. The results in Fig. 9(b) show that the dynamics of tracer cells is qualitatively similar to that calculated for the actual cells [see also Fig. 8(a) for comparison].

\section{G. Heterogeneity during tumor growth}

The effect of the glasslike or liquidlike state of tumor growth is illustrated by following the trajectories of individual cells in the growing tumor. Figures 10(a) and 10(b) highlight the trajectory of cells during a time of approximately 3 days for the average cell division time of $15 \tau_{\min }$ and $0.25 \tau_{\min }$, respectively. In the glasslike phase (intermediate times corresponding to $t / \tau<1$ for $\tau=15 \tau_{\min }$ ), the displacements are small, exhibiting caging behavior [Fig. 10(a)], resulting in the localization of the cells near their starting positions. On the other hand, cells move long 

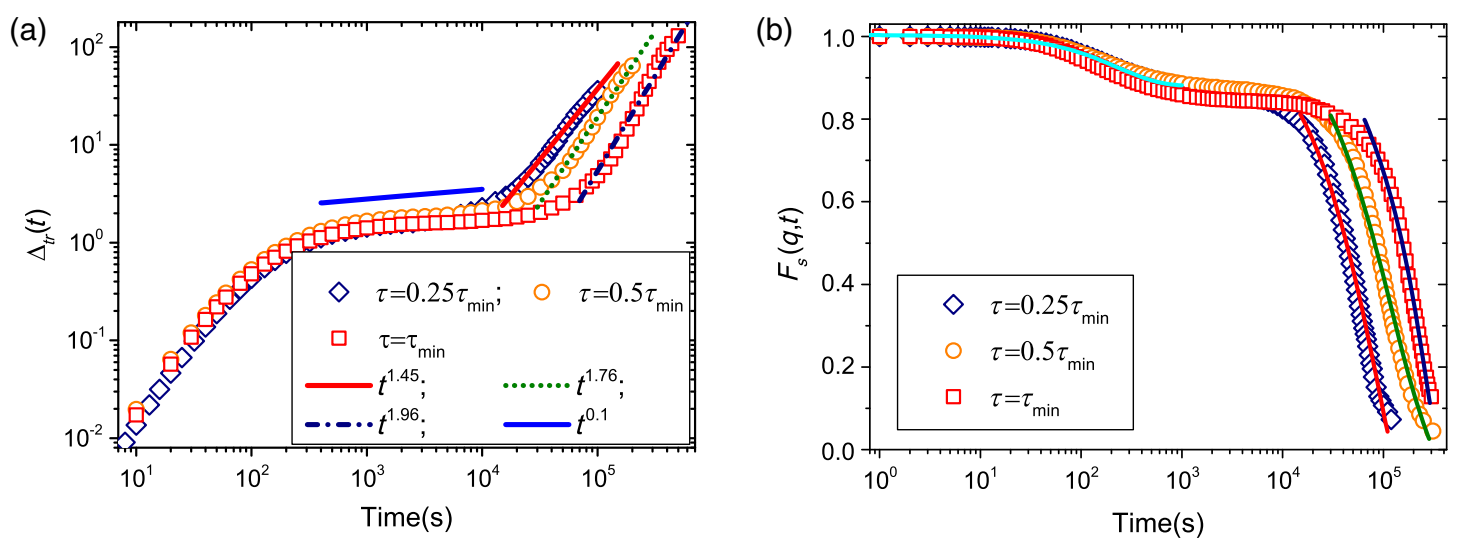

FIG. 9. Dynamics of the tracers at different cell cycle times. (a) Time dependence of $\Delta(t)$ of tracer cells at different cell division times $\tau$. Fits to $\Delta(t)$ at intermediate and long times are shown in the inset. (b) The self-intermediate scattering function $F_{s}(q, t)$ for tracers. Exponential fits to the decay of tracer $F_{s}(q, t)$ are shown by solid lines.

distances and show signatures of persistent directed motion at the shorter cell cycle time in the long time superdiffusive regime, corresponding to $t / \tau>1$ [see Fig. 10(b)]. These observations suggest that the anisotropic growth of cells, manifested largely in the evolution of cells at the periphery of the tumor, depends on the cell growth rate, a factor that determines tumor virulency.

To quantify spatial heterogeneity, we divided the tumor into two regions-interior and periphery. We note that such a division is not applicable in a system with periodic boundary conditions [27]. After obtaining the invasion distance $\Delta r\left(t=t_{E}\right)$ [see Eq. (10)], we calculated the distance from the center of mass for the colony of all the initial cells that are alive at $t_{E}$. Let us call this vector $\vec{r}_{I}$. We grouped $\vec{r}_{I}$ into two distinct categories: the interior region if $\left|\vec{r}_{I}(i)\right|<0.4 \Delta r\left(t=t_{E}\right)$ and the boundary region if $\left|\vec{r}_{I}(i)\right|>\Delta r\left(t=t_{E}\right)-2\left\langle R_{i}\right\rangle$. The average radius of all the cells in the tumor is denoted by $\left\langle R_{i}\right\rangle$. We chose $t_{E}=350000 \mathrm{~s}=6.48 \tau$. With this choice of $t_{E}$, we obtain good statistics, allowing us to glean both the subdiffusive and superdiffusive behavior from the time dependence of $\Delta(t)$ (see Fig. 11). Once the initial cells are classified in this manner, we obtained their entire trajectory history and calculated their $\Delta(t)$. In Fig. 11, a plot of $\Delta(t)$ for the interior and boundary cells is shown. The dynamics associated with interior cells is subdiffusive through their entire lifetime, while the boundary cells show subdiffusive motion at intermediate times and superdiffusive motion at long times. Interestingly, the cells at the boundary also show the intermediate glassy regime, which is a priori hard to predict.

Because the nature of cell movement determines cancer progression and metastasis [62], it is critical to understand how various factors affecting collective cell migration emerge from individual cell movements (Figs. 10 and 12). Insights into cell migrations may be obtained by using analogies to spatial heterogenous dynamics in supercooled liquids $[63,64]$. In simple fluids, the distribution of particle displacement is Gaussian, while in supercooled liquids the displacements of a subset of particles deviate from the Gaussian distribution [63]. In Fig. 12(a), the van Hove function of cell displacement (or the probability distribution of step size) is shown. The single time step distance covered by a cell is defined as $\left|\delta r_{i}(\delta t)\right|=\left|r_{i}(t+\delta t)-r_{i}(t)\right|$. By normalizing $\delta t$ by the average cell cycle time, i.e., $\delta r_{i}(\delta t / \tau=0.0074)$, we obtain a long-tailed $\delta r$ probability distribution $[P(\delta r)]$. The distribution $P(\delta r)$ has a broad, power-law tail cutoff at large values of $\delta r$, which depends on the cell cycle time. As we approach the glasslike phase for a longer average cell cycle time, $P(\delta r)$ is suppressed by an order of magnitude over a wide range of $\delta r$. Interestingly, we do not observe an abrupt change in the behavior of $P(\delta r)$ as the average cell cycle time is changed. The transition between glasslike and liquidlike regimes occurs continuously. To further analyze the displacement distribution, we fit the van Hove function for squared displacements $\left[P\left(\delta r^{2}\right)\right]$ at normal cell division time $\left(\tau_{\min }\right)$ to both the exponential and power law. The distribution is considerably broader than the Gaussian distribution (see Fig. 13 and Table II), providing one indication of heterogeneity [65].

Cell-to-cell phenotypic heterogeneity is considered to be one of the greatest challenges in cancer therapeutics $[66,67]$. Within the context of our model, spatiotemporal heterogeneity in dynamics can be observed in tissues by analyzing the movement of individual cells. While the simulated time-dependent variations in the average meansquare displacement are smooth, the movement of the individual cells is not [see Fig. 12(b)]. Cells move slowly and periodically undergo rapid "jumps" or hops similar to the phenomenon in supercooled liquids $[63,64]$. The squared displacement of individual cells [see Fig. 12(b)] vividly shows the heterogeneous behavior of different cells.

\section{H. Polarized tumor growth}

From our simulations, we constructed a spatial map of the velocities of the individual cells in the tumor. Using 
(a)
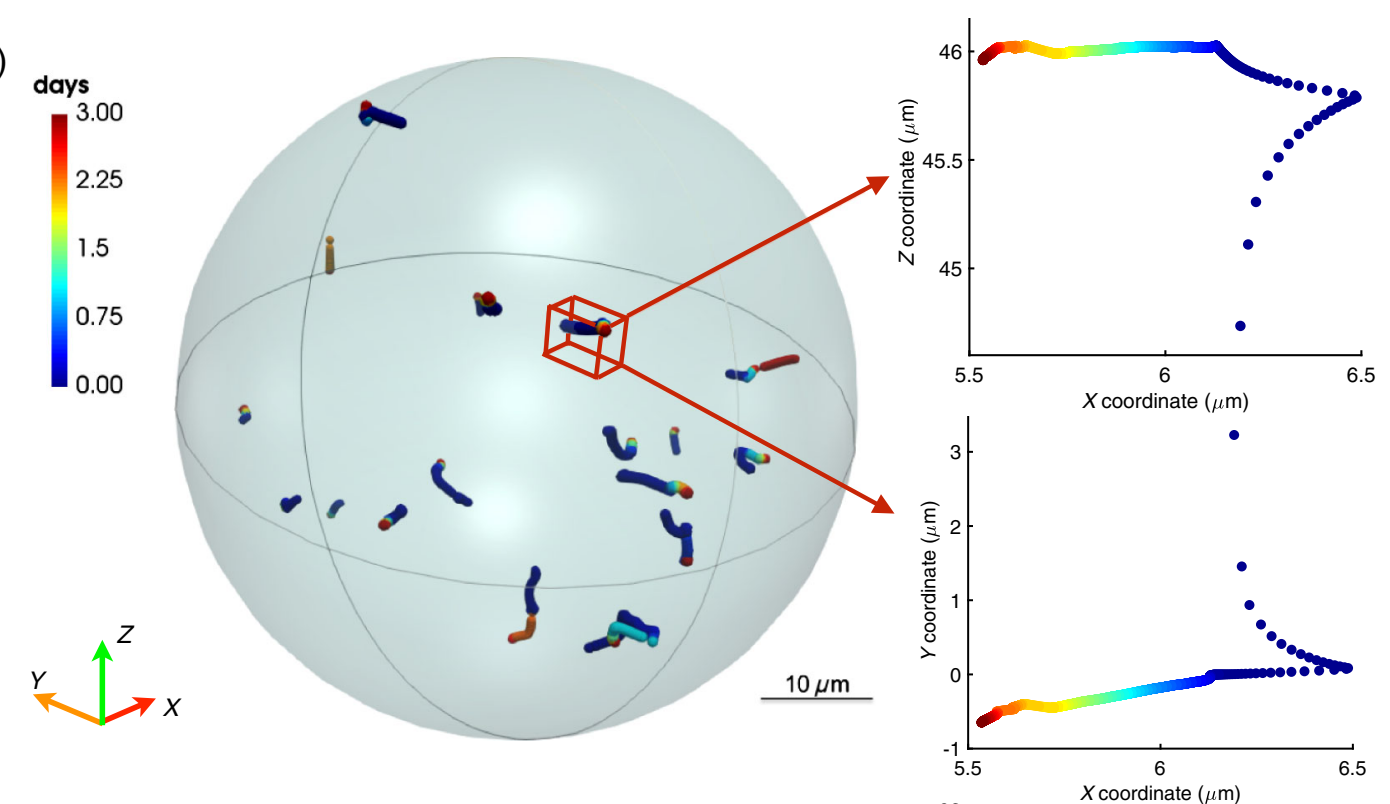

(b)
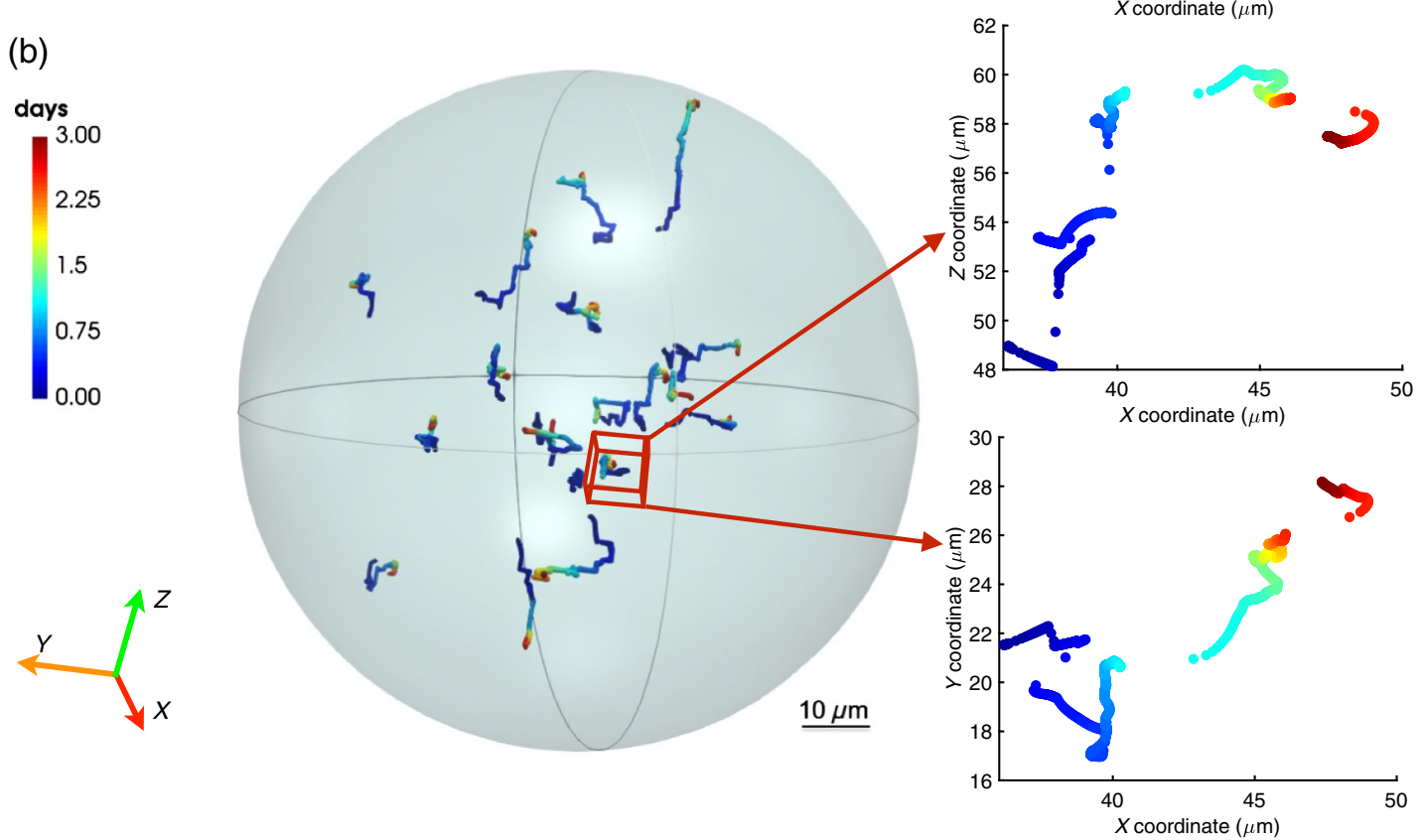

FIG. 10. Trajectories displaying spatial heterogeneity. (a) Trajectories (randomly chosen from the whole tumor) for slowly growing cells are shown. The cell cycle time is $15 \tau_{\min }$. Dynamic arrest due to caging in the glasslike phase is vividly illustrated. (b) Trajectories for rapidly growing cells with cell cycle time $\tau=0.25 \tau_{\min }$. Displacements of the cells are shown over 3 days, representing the initial stages of tumor growth in (a) and (b). Two representative trajectories (time dependence of the $x-z$ and $x-y$ coordinates) for the labeled cells are shown on the right in (a) and (b). Length in (a) and (b) is measured in units of $\mu \mathrm{m}$. The two colored spheres in (a) and (b) show the approximate extent of the tumor.

these maps, we characterized the spatial heterogeneity in the dynamics in order to elucidate regions of coordinated activity in the movement of cells. Figure 14(a) shows a snapshot of the spatial map of the single cell velocities. The velocity map, which spans more than eight orders of magnitude, reveals that there are cell-to-cell variations in the dynamics. More importantly, it also reveals the existence of spatial correlations between cell dynamics. In the tumor cross section [Fig. 14(b), Video 3], faster moving cells are concentrated at the outer periphery of the tumor. By calculating the average magnitude of cell velocity as a function of radius, we show in Fig. 14(c) that faster moving cells are located at the outer periphery of the tumor quantitatively. We calculate the average velocity of cells at different radii of the tumor using

$$
\langle v(r)\rangle=\frac{\sum_{i} v_{i} \delta\left[r-\left(\left|\vec{R}_{C M}-\vec{r}_{i}\right|\right)\right]}{\Sigma_{i} \delta\left[r-\left(\left|\vec{R}_{C M}-\vec{r}_{i}\right|\right)\right]} .
$$




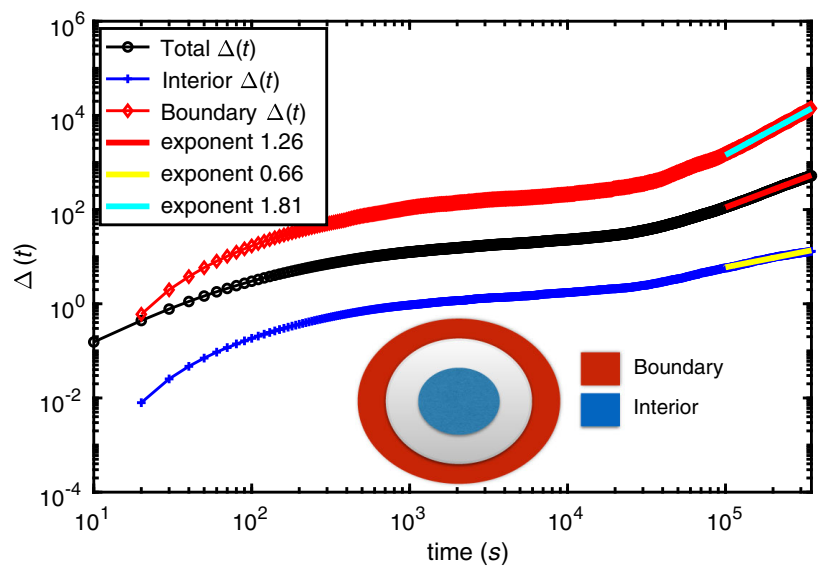

FIG. 11. Plot of the mean-square displacement $\Delta(t)$ for interior, boundary, and the total initial cells. The interior cells exhibit subdiffusive behavior through their entire lifetime. The cells at the boundary show subdiffusive motion at intermediate times, followed by superdiffusive behavior at long times. The interior $\Delta(t)$ is multiplied by 0.1 and the boundary $\Delta(t)$ by 10 for clarity. The inset shows the way we have divided the tumor into the interior and boundary regions. Here $\tau=\tau_{\min }$.
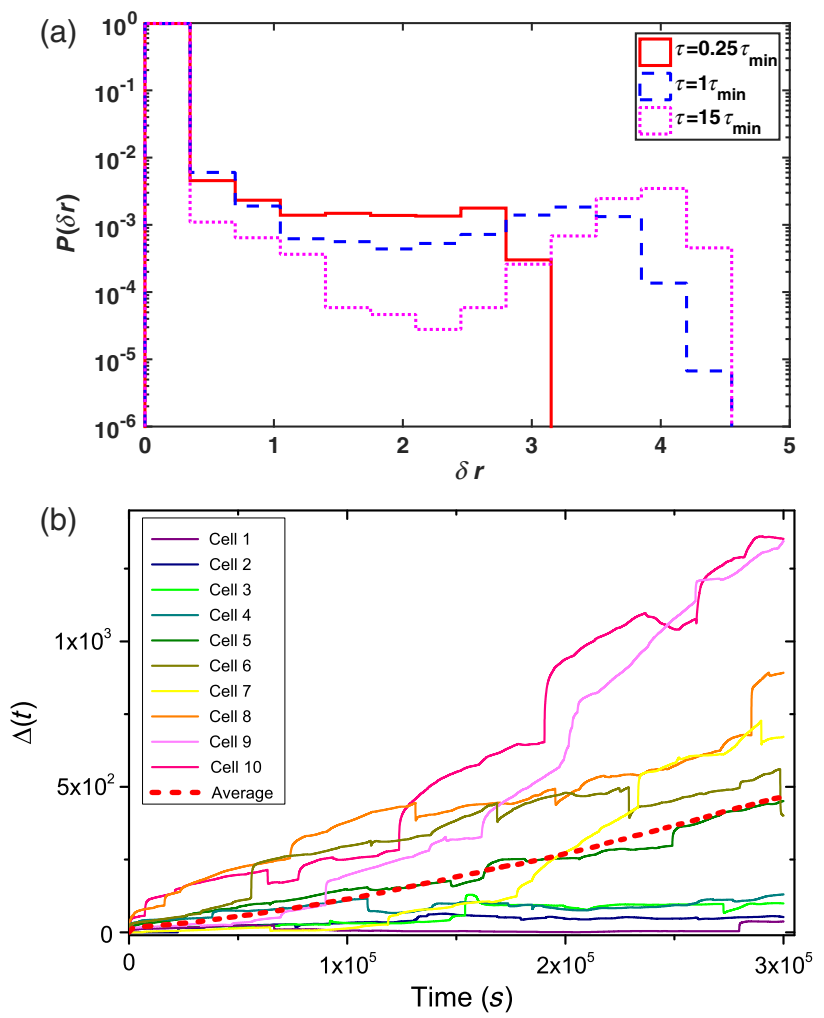

FIG. 12. Quantifying spatial heterogeneity in tumor cell growth. (a) Probability distribution of distance $\delta r$ (in units of $\mu \mathrm{m})$, moved by cells over $\delta t=100,400$, and $6000 \mathrm{~s}$, respectively, for varying average cell cycle time $\tau=0.25 \tau_{\min }, 1 \tau_{\min }$, and $15 \tau_{\min } . \delta t$ is normalized by $\tau$ to 0.0074 . (b) Time-resolved squared displacements $\Delta(t)$ (in units of $\mu \mathrm{m}^{2}$ ) of individual cells in a model for a growing tumor $\left(\tau=\tau_{\min }\right)$. The average, shown as a dashed line for approximately 800 such individual trajectories, is not meaningful because of dynamic heterogeneity.

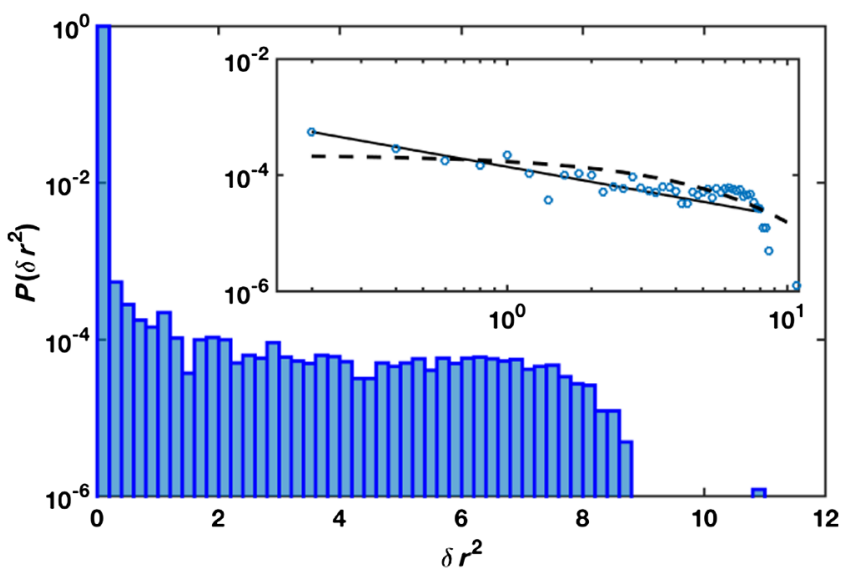

FIG. 13. The probability distribution $P\left(\delta r\left(t_{i}^{2}\right)\right)$ of cell displacements $\left[\delta r\left(t_{i}\right)^{2}=\left|r\left(t_{i}+\delta t\right)-r\left(t_{i}\right)\right|^{2}\right.$, in units of $\left.\mu \mathrm{m}^{2}\right]$ at $\delta t=$ $100 \mathrm{~s}$ is shown. Cell trajectories until $t=5 \tau$ are analyzed at $\tau=1 \tau_{\min }$. The histogram was constructed by varying $t_{i}$ in $\delta r\left(t_{i}\right)^{2}$ for over 400 cell trajectories and after obtaining approximately $10^{6}$ data points for $\delta r\left(t_{i}\right)^{2}$. For comparison, the inset shows fits to both the exponential (dashed line) and power law (solid line). $P\left(\delta r^{2}\right) \sim A \times\left(\delta r^{2}\right)^{B}$ fits the trend best, where $A$ is a constant and $B$ is about 0.9 . The striking non-Gaussian behavior, with fat power-law tails, is one indication of heterogeneity. Goodness of fit can be assessed using the parameters listed in Table II.

Arrows indicating the velocity direction show that cells in the periphery tend to move farther away from the center of the tumor as opposed to cells closer to the center of the tumor, whose direction of motion is essentially isotropic. This prediction agrees well with the experiments [33], which showed that cells at the periphery of the tumor spheroid move persistently along the radial direction, resulting in polarized tumor growth.

Mean angle $\theta$ between cell velocity and the position vector with respect to the center of the tumor plotted in Fig. 14(d) further illustrates that cell movement becomes persistently directed outward for cells closer to the outer layer of the tumor. To calculate the radius-dependent average polarization in cell velocity, we first define a vector pointing from the center of mass of the tumor to the cell position $\vec{c}_{i}=\vec{r}_{i}-\vec{R}_{C M}$ [see the green arrow in the inset of Fig. 14(d)]. The angle $\theta$ [see the inset of Fig. 14(d)] between each cell velocity (orange arrow) and the vector (green arrow) from the center of mass to the tumor periphery can be calculated from $\cos (\theta)_{i}=\vec{c}_{i} \cdot \vec{v}_{i} /\left(\left|\vec{c}_{i}\right|\left|\vec{v}_{i}\right|\right)$. The average of this angle as a function of radius is calculated using

$$
\langle\cos (\theta[r])\rangle=\frac{\Sigma_{i} \cos (\theta[r])_{i} \delta\left(r-\left(\left|\vec{R}_{C M}-\vec{r}_{i}\right|\right)\right)}{\Sigma_{i} \delta\left(r-\left(\left|\vec{R}_{C M}-\vec{r}_{i}\right|\right)\right)} .
$$

The results are presented in Fig. 14(d) in the main text. The distribution of the $\theta$ angle at different distances $(r)$ [see Fig. 15(a)] also illustrates that cell movement is isotropic 
TABLE II. The goodness of fit in the inset of Fig. 13. The $\mathrm{R}$-square value (middle column) and the root-mean-square error (RMSE) (last column) for power-law and exponential fits are provided.

\begin{tabular}{lcc}
\hline \hline Fit type & $R^{2}$ & RMSE \\
\hline Power & 0.93 & $2.4 \times 10^{-5}$ \\
Exponential & 0.57 & $5.7 \times 10^{-5}$ \\
\hline \hline
\end{tabular}

close to the tumor center, while they move outward in a directed fashion (see the peak of the histogram in blue) at the periphery of the tumor. To quantify the heterogeneity in cell velocity, we plot the probability distribution of the velocity magnitude (normalized by the mean velocity $\langle v\rangle$ ) $P(|v| /\langle v\rangle)$ [Fig. 15(b)] accessible in experiments using direct imaging or particle image velocimetry methods $[55,56]$. There is a marked change in the velocity distribution as a function of cell cycle time. At the longer cell cycle time $\left(\tau=15 \tau_{\min }\right)$, corresponding to the glasslike phase, $P(|v| /\langle v\rangle)$ distribution is clustered around smaller values of $|v| /\langle v\rangle$, while quickly decaying to zero for higher velocities.

(a)

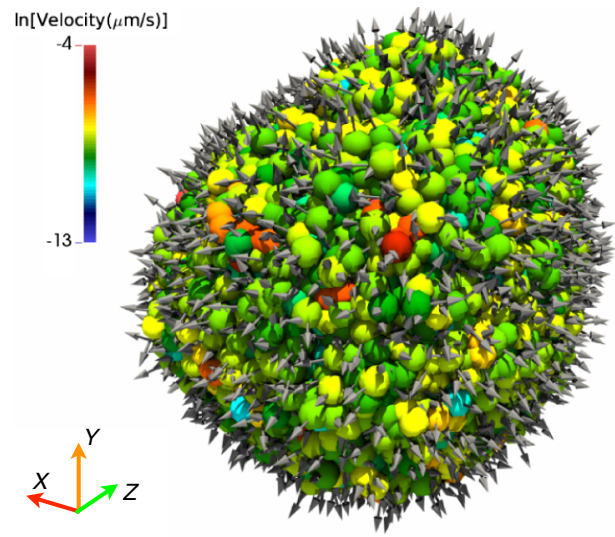

(c)

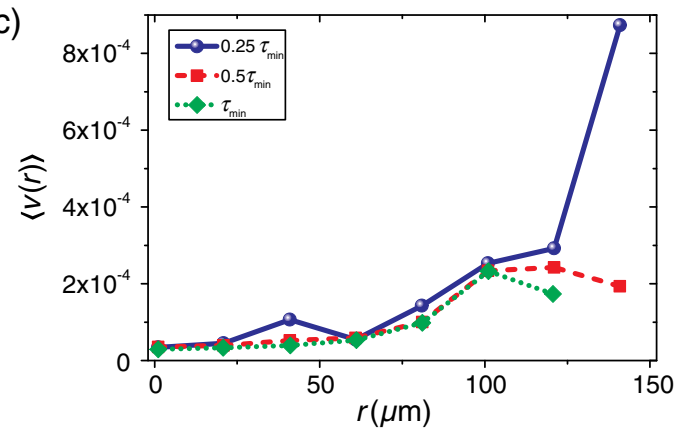

For the shorter cell cycle time, the velocity distribution is considerably broader. The broader velocity distribution indicates the presence of more invasive cells within the tumors characterized by high proliferation capacity.

\section{Consistency with experiments}

We show here that the minimal model captures the three critical aspects of a recent single-cell resolution experiment probing the invasion of cancer cells into a collagen matrix [33]: (i) Ensemble-averaged mean-square displacement of individual cells exhibits a power-law behavior at long times $\left[\Delta(t) \sim t^{\alpha}\right.$, with $\alpha \approx 1.40 \pm 0.04$ from experiments, compared with simulation results in Fig. 5(a), with $\alpha \approx 1.26 \pm 0.05$ ], indicating that, on average, directed rather than random cell motion is observed. (ii) Cells exhibit distinct topological motility profiles. At the spheroid periphery, cell movement is persistently along the radial direction, while stochastic movement is observed for cells closer to the center. Such spatial topological heterogeneity is well described as arising in our model from pressure-dependent inhibition (see Figs. 10, 12, and 14). (iii) The highly invasive spheroid boundary (deviating from

(b)

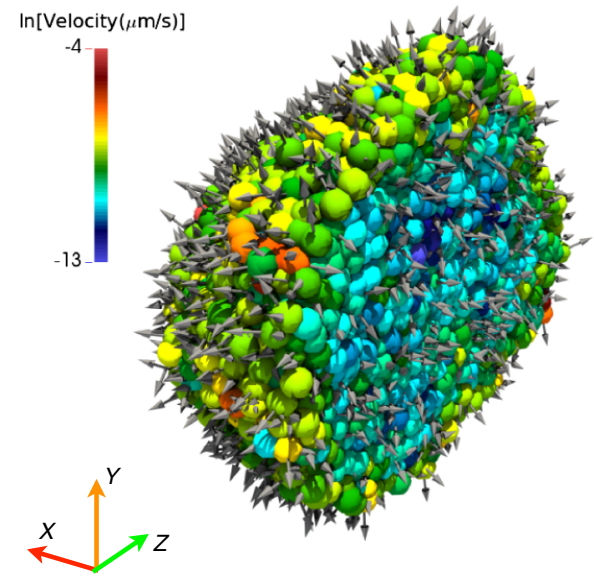

(d)

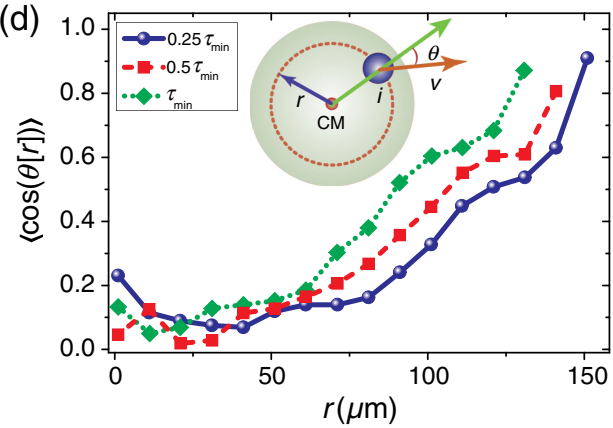

FIG. 14. Heterogeneity in the tumor cell dynamics. (a) Instantaneous snapshot of a collection $N \approx 1.3 \times 10^{4}$ cells at approximately 3 days with $\tau=0.25 \tau_{\min }$. Colors indicate the different velocities of the individual cells (in $\mu \mathrm{m} / \mathrm{s}$ ). (b) Cross section through the clump of cells shown in Fig. 14(a). Arrows denote the direction of velocity. (c) Average speed of the cells as a function of the tumor radius at different $\tau$. Observation time is at $18.5 \tau, 14.8 \tau$, and $11.1 \tau$ for $\tau=0.25 \tau_{\min }, 0.5 \tau_{\min }$, and $1 \tau_{\min }$, respectively. (d) Mean angle $\theta$ (see the inset figure) between cell velocity and a line through the center of the tumor to the periphery as a function of the tumor radius at different $\tau$. Observation time is the same as in (c). 

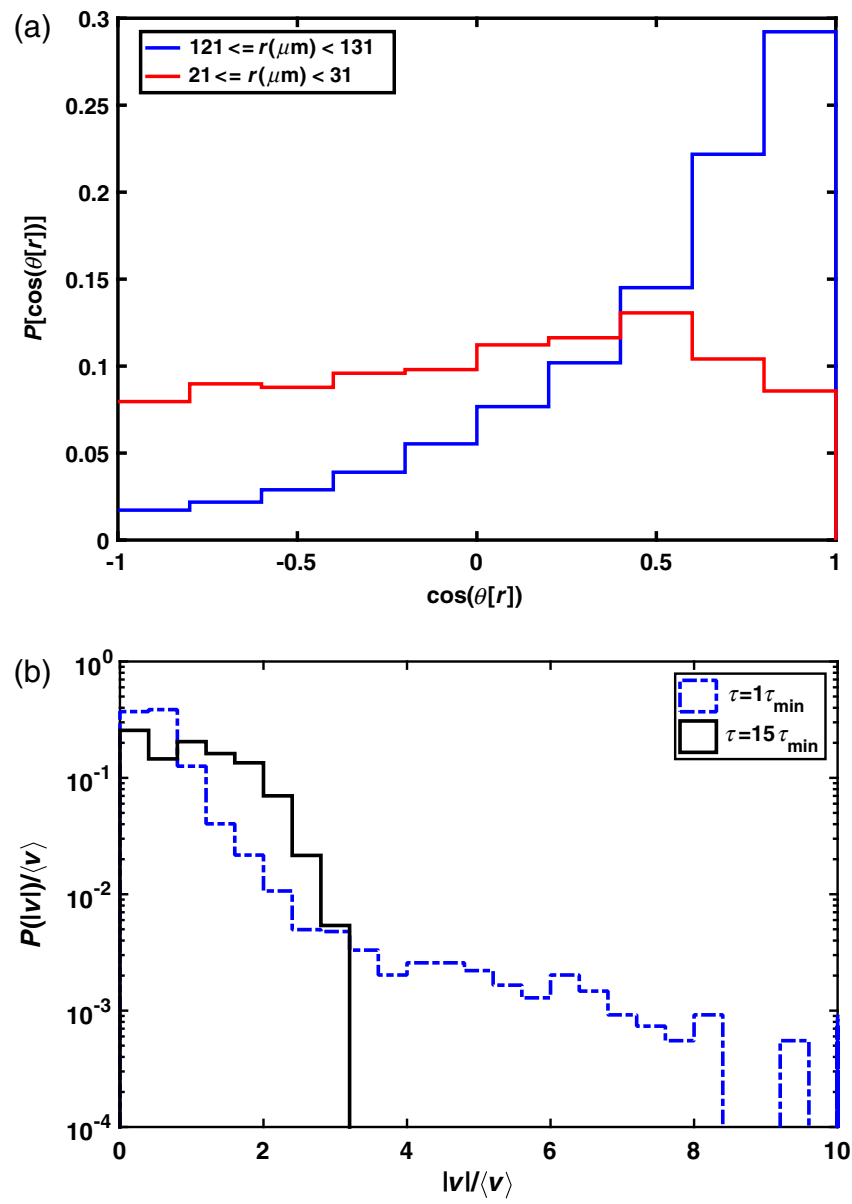

FIG. 15. Heterogeneity in the tumor cell dynamics. (a) Distribution of the angle $(\theta)$ at different distances $(r)$ from the tumor center at approximately 3 days $\left(\tau=0.25 \tau_{\min }\right)$. (b) Probability distribution of the cell speed normalized by mean cell velocity $\langle v\rangle$ at two different cell cycle times at the long time regime $(t=5 \tau)$.

what would be expected due to an isotropic random walk) as experimentally observed is qualitatively consistent with simulation results (see Fig. 6).

A salient feature of the dynamics of living cells is that birth and death processes break number conservation, having consequences on cell collective behavior [68]. To account for these processes leading to the superdiffusive behavior at long times, we establish a field theory based on stochastic quantization that accounts for the physical interactions of the cells, as well as birth and death processes. Simulations and theory suggest a mechanism of the plausible universality in the onset of superdiffusive behavior in tumor growth and unrelated systems. Remarkably, the theory predicts the dynamics of invasion at all times that are in good agreement with recent experiments [33].

\section{J. Onset of superdiffusion depends on cell division time}

In previous studies [26,27], fluidization of tissues due to cell division and apoptosis was observed at the homeostatic state. Our work shows that a glass-to-fluid transition is driven by cell division at nonsteady states and under free boundary conditions, relevant during the early stages of cancer invasion. The transition from glasslike to fluidlike behavior is determined by the average cell division time. Superdiffusion of cells in the mean-square displacement because of highly polarized tumor growth is observed on a timescale corresponding to the cell division time with a universal scaling exponent $\alpha=1.26 \pm 0.05$.

\section{K. Comparison with previous studies}

The startlingly contrasting results that we find for the dynamics of tracer cells and live cells compared to the results reported elsewhere [27] could arise for the following reasons: (i) In our simulations, we use free boundary conditions and the tumor grows continuously (with birth rate being always higher than the death rate). The effect of a free boundary is particularly pronounced at the periphery of the tumor, where the cells undergo rapid division. (ii) Imposing a birth rate that depends on the local cell density (Ref. [26]) or on the number of nearest neighbors as in Ref. [27] eventually results in a homeostatic state, where birth and death are balanced. The superdiffusive behavior observed in our study would not be present, when there is a possibility of reaching a homeostatic dense liquidlike state. In our model, this situation could be mimicked by arbitrarily increasing the cell cycle time. For example, when cell cycle times are very long, $\tau>\sim 10 \tau_{\min }$, the superdiffusive MSD exponent in the long time regime begins to deviate from approximately 1.3 to lower values [see Fig. 5(a)]. (iii) Even in our model, simple diffusion at long times is obtained if the death rate is modified. In Fig. 16, normal diffusion (red symbols) is observed at long times when the death rate is modified to

$$
k_{b}=\left[1 /\left(\tau_{\min }\right)\right] N(t) /[N(0)+N(t)],
$$

where $N(t)$ and $N(0)$ are the tumor size at time $t$ and $t=0$, respectively. At $t=0$, cells have a higher birth rate, $k_{a}=1 /\left(\tau_{\min }\right)$, compared with the death rate $\left(k_{b} / k_{a}=0.5\right)$. As the tumor grows, the death rate becomes higher, and a homeostatic state is reached once the birth and death rates are balanced, giving rise to normal diffusion, as found in Refs. [26,27]. Therefore, the superdiffusive behavior can only be found if birth and death processes are not balanced, the regime that is the focus of our study. Most importantly, if there is a mechanism for reaching homeostasis by balancing birth and death rates or making the cell division time arbitrarily long, we predict that normal diffusion would result, as shown here using Eq. (16) and previously found elsewhere [26,27].

\section{Lack of time translational invariance}

The field theory shows that the dynamics is not time translationally invariant, which is supported by 


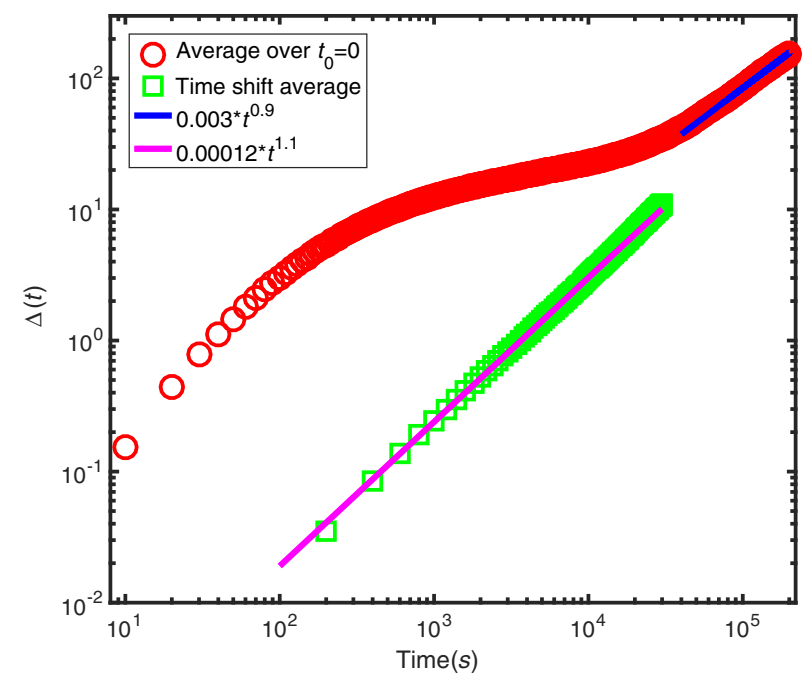

FIG. 16. The mean-square displacement $[\Delta(t)]$ of cells when cell death rate depends on time. The red circles show the results obtained by averaging over initial cell position, $\Delta(t)=\left\langle\left[r(t)-r\left(t_{0}=0\right)\right]^{2}\right\rangle$. The green squares show the results calculated using the time shift average, $\Delta(t)=\left\langle\left[r\left(t_{i}+t\right)-\right.\right.$ $\left.\left.r\left(t_{i}\right)\right]^{2}\right\rangle$. The solid lines show power-law fits to the simulation data. Normal diffusion results from the use of Eq. (16) lead to homeostasis at long times.

simulation of $\Delta(t)$. Figure 17 shows two different methods used to calculate $\Delta(t)$ : (i) the definition of MSD, $\Delta(t)=\left\langle\{1 /[N(t)]\} \Sigma_{i}\left[r_{i}(t)-r_{i}(0)\right]^{2}\right\rangle$, which always utilizes the original position of cells (at the initial simulation time) $[r(0)]$. Here, $N(t)$ is the number of initial cells and $\langle\ldots\rangle$ is the average over multiple simulation runs; (ii) $\Delta(t)=\left\langle\left[1 /\left(N_{t}\right)\right] \Sigma_{t_{1}}^{\left(t_{S}-t\right)}\left[r\left(t_{i}+t\right)-r\left(t_{i}\right)\right]^{2}\right\rangle$, a time shift average (varying $t_{i}$ ), used routinely in simulations of periodic systems with $N_{t}$ being the number of possible time intervals for a given $t$. Here, $\langle\ldots\rangle$ denotes average over initial cells. In generating the results in Fig. 17, we chose $t_{S}=500000 \mathrm{~s}$.

These two methods for computing $\Delta(t)$ produced different results, as shown in Fig. 17. The inequivalence of the two methods in obtaining $\Delta(t)$ shows that the system with free boundary conditions violates time translational invariance. The intermediate regime with subdiffusive behavior (red circles) using method (i) disappears when the second method (ii) is used to compute the $\Delta(t)$ (green squares). It is also the reason why we focused on initial cells for $\Delta(t)$ calculations. In calculating $\Delta(t)$ using the method (ii), which is commonly employed in simulations of periodic systems, a large amount of statistics is extracted for the superdiffusive regime (as it has a larger time range, on the order of approximately $10^{5} \mathrm{~s}$ ). Therefore, when we average $\Delta(t)$ over the various time intervals, the beginning time regime (which is comparatively short, approximately $10^{4} \mathrm{~s}$ ) is suppressed. It should be noted, however, that the time shift averaged method of computing $\Delta(t)$ also clearly

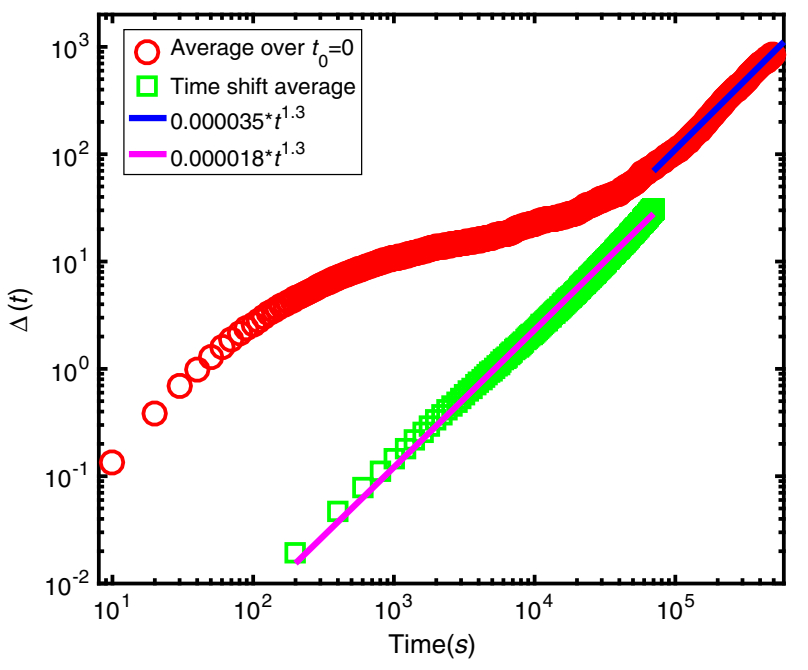

FIG. 17. Mean-square displacement $\Delta(t)$ of cells as a function of time. The red circles show the results obtained by averaging over the initial cell positions, $\Delta(t)=\left\langle[r(t)-r(0)]^{2}\right\rangle$. The green squares are the results under the time shift average, $\Delta(t)=$ $\left\langle\left[r\left(t_{i}+t\right)-r\left(t_{i}\right)\right]^{2}\right\rangle$. The solid lines show the power-law fitting of the simulation data. The long time superdiffusive behavior is evident in both plots. Here $\tau=\tau_{\min }$.

shows evidence for superdiffusive behavior over three decades of time (Fig. 17).

\section{CONCLUSIONS}

Heterogeneity is a hallmark of cancer [69]. It is difficult to capture this characteristic of cancers in well-mixed models that exclude spatial information. An important signature of cell dynamic heterogeneity-large variations in the squared displacement of cells in the tumor-is observed in our simulations. We find a broad velocity distribution among tumor cells driven by cell growth rate. The formation of spatial niches, with the tumor periphery and center as topologically distinct, is characterized by differences in proliferative and cell signaling activities. Such a distinct behavior, alluded to as the driving factor behind intratumor heterogeneity (ITH) [70,71], is not well understood. Our results predict that the pressure-dependent inhibition of cell growth is the critical factor behind the development of distinct topological niches, implying that the dynamics of cells is dependent on the microenvironment [70,71]. Cells closer to the center of the tumor spheroid are surrounded by many other cells, causing them to be predominantly in the dormant state, and they can move in random directions, while cells closer to the periphery can divide and move in a directed manner by pushing against the extracellular matrix, thus promoting tumor growth and invasiveness. We provide experimentally testable hypotheses on the signatures of heterogeneity-the onset of ITH could occur at very early stages of tumor growth (at the level of around 10000 cells). 
Although the context of our work is rooted in understanding tumor growth, we expect our model to be relevant to the study of soft glassy materials. The motion of cells in our model is surprisingly consistent with the complex motion of bubbles in a foam, also shown to be superdiffusive 36]] with the MSD exponent of $\alpha \approx 1.37 \pm 0.03$, consistent with both our theoretical predictions $(\alpha \approx 1.33)$ and simulation results $(\alpha=1.26 \pm 0.05)$. The bubbles are characterized by birth and death processes and pressure-dependent growth, which we predict to be the driving factors behind the superdiffusive behaviors observed in these diverse systems. The emergence of underlying similarities in the motion of constituent particles between living systems, such as cells, and soft glassy materials, such as foams, suggests that many of the shared, but as of yet unexplained dynamic behaviors may emerge from a common underlying theme-an imbalance in the birth and death processes and pressure-dependent growth inhibition.

\section{ACKNOWLEDGMENTS}

We acknowledge Anne D. Bowen at the Visualization Laboratory (Vislab), Texas Advanced Computing Center, for help with figure and video visualizations. We are grateful to Mauro Mugnai, Naoto Hori, and Upayan Baul for discussions and comments on the manuscript. This work was supported by the National Science Foundation through NSF Grants No. PHY 17-08128 and No. CHE 16-32756. Additional support was provided by the Welch Foundation through the Collie-Welch Chair with Grant No. F-0019.

A. N. M. and X. L. contributed equally to this work.

\section{APPENDIX A: SIMULATION TEST}

\section{Effects of random forces}

The neglect of random forces, which should be taken into account to satisfy the FDT, might seem like a drastic simplification. There are, however, two considerations. First, the tumor growth model involves birth and apoptosis. Hence, it behaves like an active system. Indeed, the theory outlined in Appendix $\mathrm{C}$ shows that, under these conditions, FDT is not satisfied, forcing us to adopt the stochastic quantization methods to compute response and correlation functions (Fig. 19). Second, from practical considerations we note that the cellular diffusion constant is $10^{-4} \mu \mathrm{m}^{2} / \mathrm{s}$ or smaller [29], resulting in only small displacements for a large fraction of cells.

In order to verify that the contributions to the dynamics arising from the random noise is small, we modified Eq. (7) to include the random forces,

$$
\dot{\vec{r}}_{i}=\frac{\vec{F}_{i}}{\gamma_{i}}+\sqrt{2 k_{B} T / \gamma_{i}^{\text {visc }}} \zeta_{i}(t)
$$

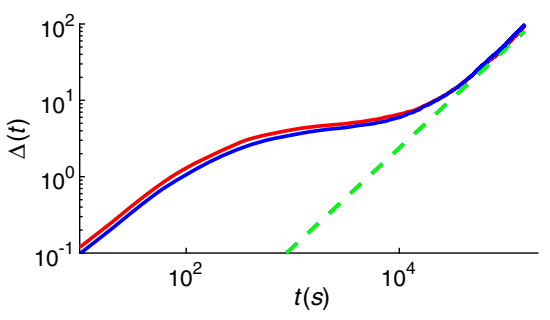

FIG. 18. Mean-square displacement $\Delta(t)$ with (blue) and without (red) random noise. The slopes obtained from the long time limit are both 1.3 (dashed green). The two curves are almost identical, thus justifying the neglect of the random noise [second term in Eq. (A1)] in the simulations.

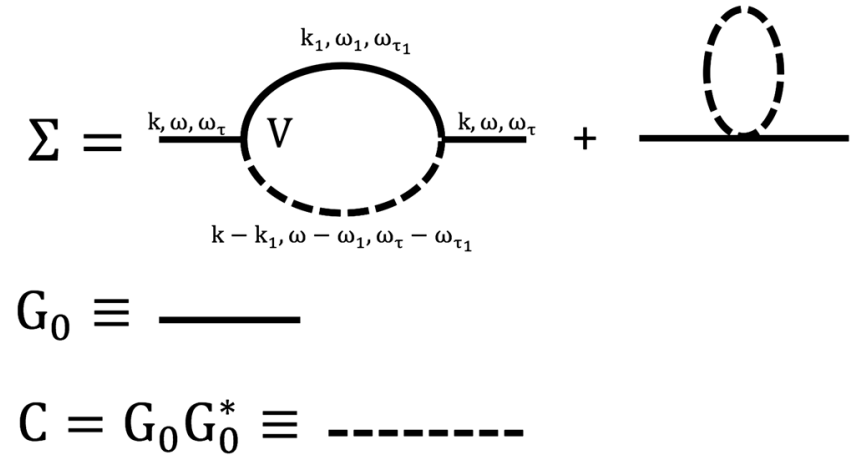

FIG. 19. The diagrams correspond to perturbation expansions of the theory [Eq. (C3)], in which the dynamical equations for the density field are expressed in fictitious time. The self-energy term $(\Sigma)$ is obtained by contracting the two density $\rho$ fields. The first diagram is the two loop contribution generated from the first order term (which contains two $\rho$ fields) in the time-dependent equation for the density fields. The second diagram, with a one loop contribution from the second order term (which contains three $\rho$ fields), resulting in the correction to $\omega^{2}+\left\{C_{0} k^{2} U(\mathbf{k})-\right.$ $\left.\left(k_{a}-2 k_{b} C_{0}\right)\right\}^{2}$, does not have any new momentum dependence. Hence, only the first term is significant in producing the scaling results.

where $k_{B}$ is the Boltzmann constant; $T$ is the temperature; and $\zeta$ is white noise with zero mean and variance, $\left\langle\zeta_{i}(t) \zeta_{i}\left(t^{\prime}\right)\right\rangle=\delta\left(t-t^{\prime}\right)$. The corresponding diffusion constant $k_{B} T / \gamma^{\text {visc }}$ is small. Thus, inclusion of random force has no consequence on the dynamics of tumor evolution. The results for $\Delta(t)$ as a function of $t$ obtained using Eqs. (7) and (A1) are identical (Fig. 18).

\section{APPENDIX B: VIDEOS}

In order to visualize the dynamic growth of the tumor, we generated videos from the simulations. They demonstrate vividly the polarized growth of the tumor, which we have quantified using various measures in the main text. 


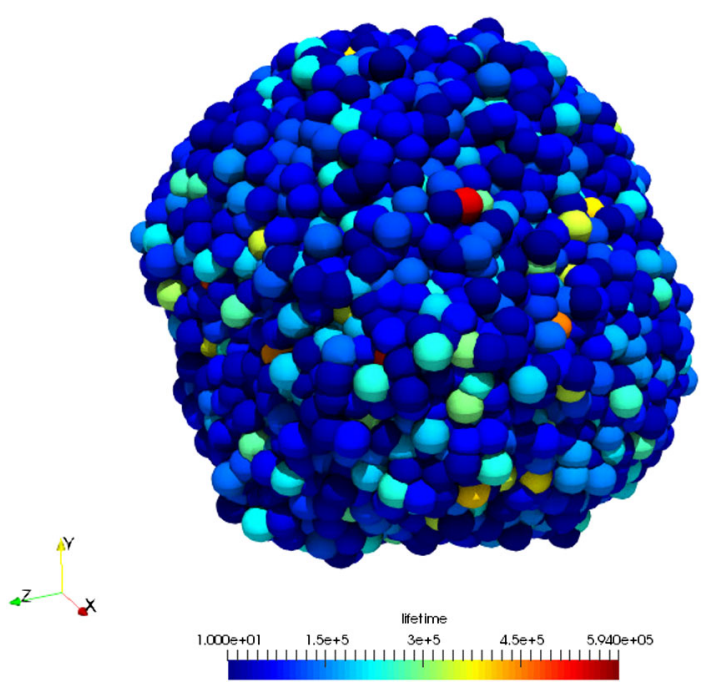

VIDEO 1. 3D growth of tumor. This video shows the threedimensional growth of the tumor over approximately 8 days. Each frame is at $1000 \mathrm{~s}$. The cell cycle time $\tau=\tau_{\min }$. The color map indicates the lifetime of the cells. Newborn cells are shown in blue and older cells that have lived longer are in red (the color bar in the video shows the cell lifetime in seconds). Cell division and death events are explicitly depicted.

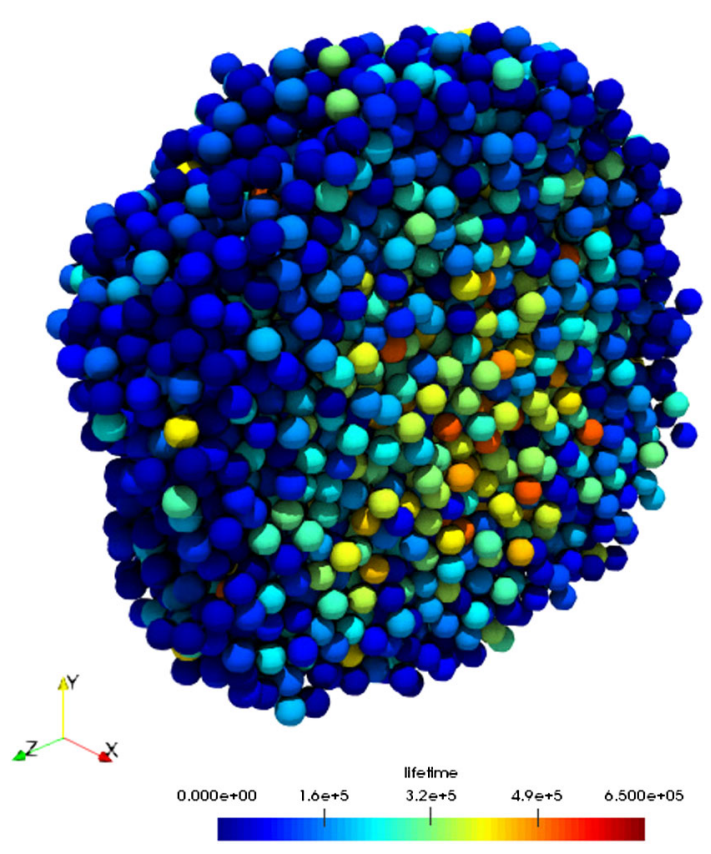

VIDEO 2. Cross section view through the growing tumor spheroid. This video illustrates an alternate view of the growing tumor shown in Video 1. Cells with longer lifetimes are mostly localized near the center of the tumor, with some of them moving to the periphery. Newly born cells are mostly located in the periphery, and division events are amplified in the periphery compared to the center of the tumor. The color bar shows the cell lifetime.

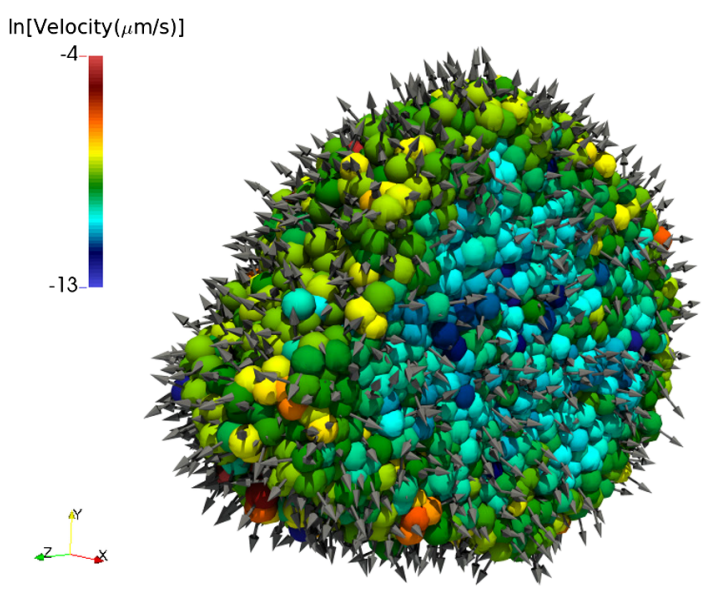

VIDEO 3. Moving clip through a tumor showing velocity heterogeneity. This video visualizes the velocity heterogeneity within the tumor. The color map indicates the speed of cells (shown in log scale) and the direction of velocity is indicated by an arrow. The video begins with a snapshot of the tumor after approximately 3 days of growth at $\tau=0.25 \tau_{\min }$. A clip moving through the tumor shows the velocity distribution of cells over different slices. It is clear that cells move slowly closer to the center, while faster moving cells are mostly in the periphery. The direction of the velocity is more randomly oriented in the tumor center but is mostly polarized outward as the periphery is approached.

\section{APPENDIX C: THEORY}

The behavior of the mean-square displacement $[\Delta(t)]$, especially the time dependence of $\Delta(t)$ at intermediate and long times, can be theoretically obtained for the tumor growth model, mimicking the one used in the simulations. We consider the dynamics of a colony of cells in a dissipative environment with negligible inertial effects. The interaction between cells is governed by adhesion and excluded volume repulsion. The equation of motion for a single cell $i$ is

$$
\frac{\partial \mathbf{r}_{i}}{\partial t}=-\sum_{j=1}^{N} \boldsymbol{\nabla} U\left[\mathbf{r}_{i}(t)-\mathbf{r}_{j}(t)\right]+\eta_{i}(t),
$$

where $U$ contains the following form of repulsive interactions with range $\lambda$ and favorable attractive interactions between cells with range $\sigma$ :

$$
\begin{aligned}
U[\mathbf{r}(i)-\mathbf{r}(j)]= & \frac{v}{\left(2 \pi \lambda^{2}\right)^{3 / 2}} e^{-\left\{\left[(\mathbf{r}(i)-\mathbf{r}(j))^{2}\right] / 2 \lambda^{2}\right\}} \\
& -\frac{\kappa}{\left(2 \pi \sigma^{2}\right)^{3 / 2}} e^{-\left\{\left[(\mathbf{r}(i)-\mathbf{r}(j))^{2}\right] / 2 \sigma^{2}\right\}} .
\end{aligned}
$$

$v$ and $\kappa$ above are the strengths of the repulsive and attractive interactions, respectively. The $\kappa$ parameter in Eq. (C2) mimics adhesion between cells. The noise $\left[\eta_{i}\right.$ in Eq. (C1)] is uncorrelated in time. 
The simplified form for $U$, which captures minimally the interactions between cells but differs from the more elaborate model used in the simulations, allows us to obtain analytical results for $\Delta(t)$ as a function of $t$. In terms of the density field of a cell, $\phi_{i}(\mathbf{r}, t)=\delta\left[\mathbf{r}-\mathbf{r}_{i}(t)\right]$, a closed form Langevin equation for the density, $\phi(\mathbf{r}, t)=\sum_{i} \phi_{i}$, can be obtained using the approach introduced by Dean [72]. In order to study tumor cell dynamics, we extend the model phenomenologically to describe both cell division and death, and we introduce a noise term that breaks the cell number conservation. These crucial features needed to describe tumor growth can be investigated using the Doi-Peliti (DP) formalism [73,74], introduced in the context of reaction-diffusion processes. A related approach was used recently by Gelimson and Golestanian [75] to describe collective dynamics in a dividing colony of chemotactic cells.

We use a scheme to study the interplay between stochastic growth and the apoptotic process, and we use it to derive a Langevin type equation for logistic growth. The birth reaction $X \stackrel{k_{a}}{\rightarrow} X+X$ occurs with the rate constant $k_{a}$ for each cell, and the backward reaction (apoptosis) $X+$ $X \stackrel{k_{b}}{\rightarrow} X$ occurs with rate $k_{b}$. By incorporating birth and apoptosis, and assuming that the density fluctuates around a constant value, $\phi(\mathbf{r}, t)=C_{0}+\rho(\mathbf{r}, t)$, we obtain the approximate equation for the density fluctuation, which in Fourier space reads

$$
\begin{aligned}
\frac{\partial \rho(\mathbf{k}, t)}{\partial t}= & -C_{0} k^{2} U(\mathbf{k}) \rho(\mathbf{k}, t)+\left(k_{a}-2 k_{b} C_{0}\right) \rho(\mathbf{k}, t) \\
& +\int d \mathbf{q}(-\mathbf{q} \cdot \mathbf{k}) U(\mathbf{q}) \rho(\mathbf{q}, t) \rho(\mathbf{k}-\mathbf{q}, t) \\
& -k_{b} \int d \mathbf{q} \rho(\mathbf{q}, t) \rho(\mathbf{k}-\mathbf{q})+\sqrt{k_{a} C_{0}+k_{b} C_{0}^{2}} f_{\phi} .
\end{aligned}
$$

We derived Eq. (C3) by expanding the density to lowest order in $\rho / C_{0}$ nonlinearity. The noise $f_{\phi}$ satisfies $\left\langle f_{\phi}(\mathbf{r}, t) f_{\phi}\left(\mathbf{r}^{\prime}, t^{\prime}\right)\right\rangle=\delta\left(\mathbf{r}-\mathbf{r}^{\prime}\right) \delta\left(t-t^{\prime}\right)$. In the hydrodynamic, $k \rightarrow 0$ and $t \rightarrow \infty$ limit, the first and third terms in the rhs of Eq. (C3) vanish and, hence, the scaling behavior of $\Delta(t)$ at long times is determined solely by the death-birth terms.

The scaling of $\Delta(t)$ can be obtained by treating the nonlinear terms in Eq. (C3) perturbatively using the Parisi$\mathrm{Wu}$ stochastic quantization scheme $[59,76,77]$, which is needed because the FDT is not satisfied in Eq. (C3) because of the cell birth and death processes. In order to outline the essence of the theory, let us consider the probability distribution corresponding to the noise term, given by
$P\left(f_{\phi}\right)(\mathbf{k}, \omega) \propto \exp \left[-\int \frac{d^{D} \mathbf{k}}{(2 \pi)^{D}} \frac{d \omega}{2 \pi} \frac{1}{2} f_{\phi}(\mathbf{k}, \omega) f_{\phi}(-\mathbf{k},-\omega)\right]$.

By reexpressing $P\left(f_{\phi}(\mathbf{k}, \omega)\right)$ in terms of $P(\rho(\mathbf{k}, \omega))$, a Langevin equation of motion in the fictitious time $\tau_{f}$ may be derived, in which FDT is satisfied. Consequently, in the $\tau_{f} \rightarrow \infty$ limit, the distribution function $P(\rho(\mathbf{k}, \omega)) \propto$ $\exp (-S(\mathbf{k}, \omega))$, where an expression for the effective action $S(\mathbf{k}, \omega)$ is derivable from Eqs. (C3) and (C4). Using this formalism, the Green's function can be obtained using perturbation theory by solving the Dyson equation,

$$
[G]^{-1}=\left[G^{(0)}\right]^{-1}+\Sigma\left(\mathbf{k}, \omega, \omega_{\tau_{f}}\right),
$$

where $\omega_{\tau_{f}}$ is the frequency related to $\tau_{f}$ and $G_{0}^{-1}=i \omega_{\tau_{f}}+$ $\left\{1 /\left[2\left(k_{a} C_{0}+k_{b} C_{0}^{2}\right)\right]\right\}\left(\omega^{2}+\left\{C_{0} k^{2} U(\mathbf{k})-\left(k_{a}-2 k_{b} C_{0}\right)\right\}^{2}\right)$. A diagrammatic representation of the self-energy term $\Sigma\left(\mathbf{k}, \omega, \omega_{\tau_{f}}\right)$ is shown in Fig. 19 to one loop order. We obtain $\Sigma\left(\mathbf{k}, \omega, \omega_{\tau_{f}}\right) \sim \int\left\{\left(d^{D} \mathbf{k}_{\mathbf{1}}\right) /\left[(2 \pi)^{D}\right]\right\}\left[\left(d \omega_{1}\right) /(2 \pi)\right]$ $\left[\left(d \omega_{\tau_{f 1}}\right) /(2 \pi)\right] V V G_{0} C_{0}$, where the vertex term is of the form $V=\left\{1 /\left[2\left(k_{a} C_{0}+k_{b} C_{0}^{2}\right)\right]\right\}\left(\left\{i \omega+C_{0} k^{2} U(\mathbf{k})-\right.\right.$ $\left.\left(k_{a}-2 k_{b} C_{o}\right)\right\}\left\{\left(-\mathbf{k}_{\mathbf{1}} \cdot \mathbf{k}\right) U\left(\mathbf{k}_{\mathbf{1}}\right)-k_{b}\right\}+\left\{i \omega_{1}+C_{0} k_{1}^{2} U\left(\mathbf{k}_{\mathbf{1}}\right)-\right.$ $\left.\left(k_{a}-2 k_{b} C_{o}\right)\right\}\left\{\left(-\mathbf{k}_{\mathbf{1}} \cdot \mathbf{k}\right) U(-\mathbf{k})-k_{b}\right\}+\left\{i \omega_{1}+C_{0} k_{1}^{2} U\left(\mathbf{k}_{\mathbf{1}}\right)-\right.$ $\left.\left.\left(k_{a}-2 k_{b} C_{o}\right)\right\}\left\{\left(-\mathbf{k}_{\mathbf{1}} \cdot\left(\mathbf{k}-\mathbf{k}_{\mathbf{1}}\right)\right) U\left(\mathbf{k}-\mathbf{k}_{\mathbf{1}}\right)-k_{b}\right\}\right)$, the correlation $C_{0}=G_{0} G_{0}^{*}$, and $D$ is the spatial dimension. After computing the self-energy to second order in nonlinearity, Eq. (C5) can be written as

$$
[G]^{-1}\left(\mathbf{k}, \omega, \omega_{\tau_{f}}\right)=-i \omega_{\tau_{f}}+\frac{1}{2\left(D_{0}\right)}\left[\omega^{2}\right]+\frac{1}{2(\bar{D})}\left[\nu_{\mathrm{eff}}^{2} k^{4}\right],
$$

where $D_{0}=k_{a} C_{0}+k_{b} C_{0}^{2}$. The above equation allows us to determine an effective coefficient $\bar{D}$ from $G^{-1}(\mathbf{k}, 0,0)$,

$$
\frac{1}{2(\bar{D})}\left[\nu_{\mathrm{eff}}^{2} k^{4}\right]=\frac{1}{2\left(D_{0}\right)}\left(\nu k^{2}\right)^{2}+\Sigma\left(\mathbf{k}, \omega, \omega_{\tau_{f}}\right),
$$

with $\nu=C_{0} U(\mathbf{k})$. In obtaining Eq. (C7), needed for calculating the scaling of $\Delta(t)$ in the intermediate time, the strength of the interactions are such that $C_{0} k^{2} U(\mathbf{k})$ dominates over $\left(k_{a}-2 k_{b} C_{0}\right)$. Expanding $\nu_{\text {eff }}$ about $\nu$ and $\bar{D}$ around $D_{0}$, and noting that the renormalization of $\nu$ dominates, we write, using $\Delta \nu=\nu_{\text {eff }}-\nu$,

$$
\Delta \nu k^{2}=\frac{1}{2 \nu k^{2}} \Sigma\left(\mathbf{k}, \omega, \omega_{\tau_{f}}\right) .
$$

In the spirit of a self-consistent mode coupling theory, we now replace $\nu$ by $\Delta \nu$ in the self-energy term $\Sigma\left(\mathbf{k}, \omega, \omega_{\tau_{f}}\right)$, and we use $G$, as given by Eq. (C6), and the correlation function $C=G G^{*}$, as follows from the FDT. According to 
scale transformation, $\omega \sim k^{z}, \omega_{\tau_{f}} \sim k^{2 z}, G \sim k^{-2 z}, C \sim k^{-4 z}$, and the vertex factor $V \sim k^{z+2}$. The self-energy term in Fig. (19) can be written as $\Sigma\left(\mathbf{k}, \omega, \omega_{\tau_{f}}\right) \sim \int\left\{\left(d^{D} \mathbf{k}^{\prime}\right) /\right.$ $\left.\left[(2 \pi)^{D}\right]\right\}\left[\left(d \omega^{\prime}\right) /(2 \pi)\right]\left[\left(d \omega_{\tau_{f}}^{\prime}\right) /(2 \pi)\right] V V G C$ (Fig. 19 provides a diagrammatic representation of the theory). By carrying out the momentum count of $\Sigma\left(\mathbf{k}, \omega, \omega_{\tau_{f}}\right)$, and noting that $\nu k^{2} \sim k^{z}$, we find $\Sigma\left(k, \omega, \omega_{\tau_{f}}\right) \sim k^{D-z+4}$. Using Eq. (C8), we obtain $k^{z+2} \sim k^{D-z+4}$, leading to $z=1+(D / 2)$.

The scaling of $\Delta(t)$ at intermediate and long times may be gleaned using the relation $C=\left(1 / \omega_{\tau_{f}}\right) \operatorname{Im} G$. Assuming dynamic scaling holds, the single cell mean-square displacement should behave as

$$
\Delta(t)=\left\langle[\mathbf{r}(t)-\mathbf{r}(0)]^{2}\right\rangle \sim t^{2 / z}=t^{\alpha} .
$$

In $3 \mathrm{D}, \alpha=\frac{4}{5}=0.8$, implying that $\Delta(t)$ should display subdiffusive behavior. The theoretical prediction is in accord with the behavior of $\Delta(t)$ in the caging regime. In the long time limit, the nonlinearity due to death-birth dominates over mechanical interactions $[\propto U(\mathbf{k})]$. A similar procedure, as mentioned above, produces the dynamic exponent $z=D / 2$. In this regime, $\alpha=1.33$, implying superdiffusive motion, a prediction that is also in agreement with our simulations and experimental results [33]. Thus, the theory explains the simulation results, and by extension the experimental data, nearly quantitatively.

[1] B. A. Barres, I. K. Hart, H. S. R. Coles, J. F. Burne, J. T. Voyvodic, W. D. Richardson, and M. C. Raff, Cell Death and Control of Cell Survival in the Oligodendrocyte Lineage, Cell 70, 31 (1992).

[2] R. Weinberg, The Biology of Cancer (Garland Science, New York, 2013).

[3] D. Ingber, Mechanobiology and Diseases of Mechanotransduction, Ann. Med. 35, 564 (2003).

[4] F. Guilak, D. M. Cohen, B. T. Estes, J. M. Gimble, W. Liedtke, and C.S. Chen, Control of Stem Cell Fate by Physical Interactions with the Extracellular Matrix, Cell Stem Cell 5, 17 (2009).

[5] S. Kumar and V. M. Weaver, Mechanics, Malignancy, and Metastasis: The Force Journey of a Tumor Cell, Cancer Metastasis Rev. 28, 113 (2009).

[6] W. G. Stetler-Stevenson, S. Aznavoorian, and L. A. Liotta, Tumor Cell Interactions with the Extracellular Matrix During Invasion and Metastasis, Annual Review of Cell Biology 9, 541 (1993).

[7] D. T. Tambe, C. C. Hardin, T. E. Angelini, K. Rajendran, C. Y. Park, X. Serra-Picamal, E. H. Zhou, M. H. Zaman, J. P. Butler, D. A. Weitz et al., Collective Cell Guidance by Cooperative Intercellular Forces, Nat. Mater. 10, 469 (2011).

[8] M. C. Marchetti, J. F. Joanny, S. Ramaswamy, T. B. Liverpool, J. Prost, M. Rao, and R. A. Simha, Hydrodynamics of Soft Active Matter, Rev. Mod. Phys. 85, 1143 (2013).
[9] T. E. Angelini, E. Hannezo, X. Trepat, M. Marquez, J. J. Fredberg, and D. A. Weitz, Glass-Like Dynamics of Collective Cell Migration, Proc. Natl. Acad. Sci. U.S.A. 108, 4714 (2011).

[10] M. Sadati, N. T. Qazvini, R. Krishnan, C. Y. Park, and J. J. Fredberg, Collective Migration and Cell Jamming, Differentiation (Berlin) 86, 121 (2013).

[11] T. E. Angelini, E. Hannezo, X. Trepat, J. J. Fredberg, and D. A. Weitz, Cell Migration Driven by Cooperative Substrate Deformation Patterns, Phys. Rev. Lett. 104, 168104 (2010).

[12] E.-M. Schoetz, M. Lanio, J. A. Talbot, and M. L. Manning, Glassy dynamics in three-dimensional embryonic tissues, J. R. Soc. Interface 10, 20130726 (2013).

[13] I. González-García, R. V. Solé, and J. Costa, Metapopulation Dynamics and Spatial Heterogeneity in Cancer, Proc. Natl. Acad. Sci. U.S.A. 99, 13085 (2002).

[14] R. Durrett and S. A. Levin, Stochastic Spatial Models: A User's Guide to Ecological Applications, Phil. Trans. R. Soc. B 343, 329 (1994).

[15] T. Nagai and H. Honda, A Dynamic Cell Model for the Formation of Epithelial Tissues, Philos. Mag. B 81, 699 (2001).

[16] D. Bi, X. Yang, M. C. Marchetti, and M. L. Manning, Motility-Driven Glass and Jamming Transitions in Biological Tissues, Phys. Rev. X 6, 021011 (2016).

[17] R. Farhadifar, J.-C. Röper, B. Aigouy, S. Eaton, and F. Jülicher, The Influence of Cell Mechanics, Cell-Cell Interactions, and Proliferation on Epithelial Packing, Curr. Biol. 17, 2095 (2007).

[18] B. Li and S. X. Sun, Coherent Motions in Confluent Cell Monolayer Sheets, Biophys. J. 107, 1532 (2014).

[19] A. G. Fletcher, M. Osterfield, R. E. Baker, and S. Y. Shvartsman, Vertex Models of Epithelial Morphogenesis, Biophys. J. 106, 2291 (2014).

[20] T. J. Newman, Modeling Multi-Cellular Systems Using Subcellular Elements, Math. Biosci. Eng. 2, 613 (2005).

[21] F. Graner and J. A. Glazier, Simulation of Biological Cell Sorting Using a Two-Dimensional Extended Potts Model, Phys. Rev. Lett. 69, 2013 (1992).

[22] A. Szabó, R. Ünnep, E. Méhes, W. O. Twal, W. S. Argraves, Y. Cao, and A. Czirók, Collective Cell Motion in Endothelial Monolayers, Phys. Biol. 7, 046007 (2010).

[23] B. A. Camley, Y. Zhang, Y. Zhao, B. Li, E. Ben-Jacob, H. Levine, and W.-J. Rappel, Polarity Mechanisms Such as Contact Inhibition of Locomotion Regulate Persistent Rotational Motion of Mammalian Cells on Micropatterns, Proc. Natl. Acad. Sci. U.S.A. 111, 14770 (2014).

[24] B. A. Camley and W.-J. Rappel, Physical Models of Collective Cell Motility: From Cell to Tissue, J. Phys. D 50, 113002 (2017).

[25] S. Hilgenfeldt, S. Erisken, and R. W. Carthew, Physical Modeling of Cell Geometric Order in an Epithelial Tissue, Proc. Natl. Acad. Sci. U.S.A. 105, 907 (2008).

[26] J. Ranft, M. Basan, J. Elgeti, J.-F. Joanny, J. Prost, and F. Jülicher, Fluidization of Tissues by Cell Division and Apoptosis, Proc. Natl. Acad. Sci. U.S.A. 107, 20863 (2010).

[27] D. A. Matoz-Fernandez, K. Martens, R. Sknepnek, J. L. Barrat, and S. Henkes, Cell Division and Death Inhibit Glassy Behaviour of Confluent Tissues, Soft Matter 13, 3205 (2017). 
[28] D. Drasdo and S. Höhme, A Single-Cell-Based Model of Tumor Growth In Vitro: Monolayers and Spheroids, Phys. Biol. 2, 133 (2005).

[29] G. Schaller and M. Meyer-Hermann, Multicellular Tumor Spheroid in an Off-Lattice Voronoi-Delaunay Cell Model, Phys. Rev. E 71, 051910 (2005).

[30] P.-H. Wu, A. Giri, S.X. Sun, and D. Wirtz, ThreeDimensional Cell Migration Does Not Follow a Random Walk, Proc. Natl. Acad. Sci. U.S.A. 111, 3949 (2014).

[31] N. Gal and D. Weihs, Intracellular Mechanics and Activity of Breast Cancer Cells Correlate with Metastatic Potential, Cell Biochem. Biophys. 63, 199 (2012).

[32] P. Dieterich, R. Klages, R. Preuss, and A. Schwab, Anomalous Dynamics of Cell Migration, Proc. Natl. Acad. Sci. U.S.A. 105, 459 (2008).

[33] A. M. J. Valencia, P.-H. Wu, O. N. Yogurtcu, P. Rao, J. DiGiacomo, I. Godet, L. He, M.-H. Lee, D. Gilkes, S. X. Sun et al., Collective Cancer Cell Invasion Induced by Coordinated Contractile Stresses, Oncotarget 6, 43438 (2015).

[34] M. Pickl and C. H. Ries, Comparison of 3D and 2D Tumor Models Reveals Enhanced HER2 Activation in 3D Associated with an Increased Response to Trastuzumab, Oncogene 28, 461 (2009).

[35] K. Wolf, Y. I. Wu, Y. Liu, J. Geiger, E. Tam, C. Overall, M. S. Stack, and P. Friedl, Multi-Step Pericellular Proteolysis Controls the Transition from Individual to Collective Cancer Cell Invasion, Nat. Cell Biol. 9, 893 (2007).

[36] H. J. Hwang, R. A. Riggleman, and J. C. Crocker, Understanding Soft Glassy Materials Using an Energy Landscape Approach, Nat. Mater. 15, 1031 (2016).

[37] P. Pathmanathan, J. Cooper, A. Fletcher, G. Mirams, P. Murray, J. Osborne, J. Pitt-Francis, A. Walter, and S. J. Chapman, A Computational Study of Discrete Mechanical Tissue Models, Phys. Biol. 6, 036001 (2009).

[38] E. Palsson and H. G. Othmer, A Model for Individual and Collective Cell Movement in Dictyostelium Discoideum, Proc. Natl. Acad. Sci. U.S.A. 97, 10448 (2000).

[39] J. C. Dallon and H. G. Othmer, How Cellular Movement Determines the Collective Force Generated by the Dictyostelium Discoideum Slug, J. Theor. Biol. 231, 203 (2004).

[40] E. Palsson, A Three-Dimensional Model of Cell Movement in Multicellular Systems, Future Gener. Comput. Syst. 17, 835 (2001).

[41] D. Drasdo and S. Höhme, Individual-Based Approaches to Birth and Death in Avascular Tumors, Math. Comput. Modell. 37, 1163 (2003).

[42] F. Montel, M. Delarue, J. Elgeti, L. Malaquin, M. Basan, T. Risler, B. Cabane, D. Vignjevic, J. Prost, G. Cappello et al., Stress Clamp Experiments on Multicellular Tumor Spheroids, Phys. Rev. Lett. 107, 188102 (2011).

[43] G. Helmlinger, P. A. Netti, H. C. Lichtenbeld, R. J. Melder, and R. K. Jain, Solid Stress Inhibits the Growth of Multicellular Tumor Spheroids, Nat. Biotechnol. 15, 778 (1997).

[44] J. Galle, M. Loeffler, and D. Drasdo, Modeling the Effect of Deregulated Proliferation and Apoptosis on the Growth Dynamics of Epithelial Cell Populations In Vitro, Biophys. J. 88, 62 (2005).

[45] J. P. Freyer and R. M. Sutherland, Regulation of Growth Saturation and Development of Necrosis in EMT6/Ro
Multicellular Spheroids by the Glucose and Oxygen Supply, Cancer Res. 46, 3504 (1986).

[46] J. J. Casciari, S. V. Sotirchos, and R. M. Sutherland, Variations in Tumor Cell Growth Rates and Metabolism with Oxygen Concentration, Glucose Concentration, and Extracellular pH, J. Cell. Physiol. 151, 386 (1992).

[47] J. Landry, J. P. Freyer, and R. M. Sutherland, Shedding of Mitotic Cells from the Surface of Multicell Spheroids During Growth, J. Cell. Physiol. 106, 23 (1981).

[48] D. F. Quail and J. A. Joyce, Microenvironmental Regulation of Tumor Progression and Metastasis, Nat. Med. 19, 1423 (2013).

[49] R. K. Jain, J. D. Martin, and T. Stylianopoulos, The Role of Mechanical Forces in Tumor Growth and Therapy, Annu. Rev. Biomed. Eng. 16, 321 (2014).

[50] A. D. Conger and M. C. Ziskin, Growth of Mammalian Multicellular Tumor Spheroids, Cancer Res. 43, 556 (1983).

[51] E. Mandonnet, J.-Y. Delattre, M.-L. Tanguy, K. R. Swanson, A. F. Carpentier, H. Duffau, P. Cornu, R. V. Effenterre, E. C. Alvord, and L. Capelle, Continuous Growth of Mean Tumor Diameter in a Subset of Grade II Gliomas, Annals of Neurology 53, 524 (2003).

[52] M. Simeoni, P. Magni, C. Cammia, G. De Nicolao, V. Croci, E. Pesenti, M. Germani, I. Poggesi, and M. Rocchetti, Predictive Pharmacokinetic-Pharmacodynamic Modeling of Tumor Growth Kinetics in Xenograft Models after Administration of Anticancer Agents, Cancer Res. 64, 1094 (2004).

[53] D. Hart, E. Shochat, and Z. Agur, The Growth Law of Primary Breast Cancer as Inferred from Mammography Screening Trials Data, Br. J. Cancer 78, 382 (1998).

[54] D. R. Grimes, P. Kannan, A. McIntyre, A. Kavanagh, A. Siddiky, S. Wigfield, A. Harris, and M. Partridge, The Role of Oxygen in Avascular Tumor Growth, PLoS One 11, e0153692 (2016).

[55] F. Pampaloni, E. G. Reynaud, and E. H. K. Stelzer, The Third Dimension Bridges the Gap Between Cell Culture and Live Tissue, Nat. Rev. Mol. Cell Biol. 8, 839 (2007).

[56] K. Alessandri, B. R. Sarangi, V. V. Gurchenkov, B. Sinha, T. R. Kießling, L. Fetler, F. Rico, S. Scheuring, C. Lamaze, A. Simon et al., Cellular Capsules as a Tool for Multicellular Spheroid Production and for Investigating the Mechanics of Tumor Progression In Vitro, Proc. Natl. Acad. Sci. U.S.A. 110, 14843 (2013).

[57] E. R. Weeks, J. C. Crocker, A. C. Levitt, A. Schofield, and D. A. Weitz, Three-Dimensional Direct Imaging of Structural Relaxation near the Colloidal Glass Transition, Science 287, 627 (2000).

[58] W. K. Kegel and A. van Blaaderen, Direct Observation of Dynamical Heterogeneities in Colloidal Hard-Sphere Suspensions, Science 287, 290 (2000).

[59] G. Parisi and Y. S. Wu, Perturbation Theory without Gauge Fixing, Scientia Sinica 24, 483 (1981).

[60] T. R. Kirkpatrick, D. Thirumalai, and P. G. Wolynes, Scaling Concepts for the Dynamics of Viscous Liquids near an Ideal Glassy State, Phys. Rev. A 40, 1045 (1989).

[61] L. Berthier, G. Biroli, J.-P. Bouchaud, L. Cipelletti, and W. van Saarloos, Dynamical Heterogeneities in Glasses, Colloids, and Granular Media (OUP, Oxford, 2011), Vol. 150. 
[62] P. Friedl and D. Gilmour, Collective Cell Migration in Morphogenesis, Regeneration and Cancer, Nat. Rev. Mol. Cell Biol. 10, 445 (2009).

[63] D. Thirumalai and R. D. Mountain, Activated Dynamics, Loss of Ergodicity, and Transport in Supercooled Liquids, Phys. Rev. E 47, 479 (1993).

[64] J.-L. Barrat, J.-N. Roux, and J.-P. Hansen, Diffusion, Viscosity and Structural Slowing down in Soft Sphere Alloys near the Kinetic Glass Transition, Chem. Phys. 149, 197 (1990).

[65] T. R. Kirkpatrick and D. Thirumalai, Colloquium: Random First Order Transition Theory Concepts in Biology and Physics, Rev. Mod. Phys. 87, 183 (2015).

[66] M. D. Brooks, M. L. Burness, and M. S. Wicha, Therapeutic Implications of Cellular Heterogeneity and Plasticity in Breast Cancer, Cell Stem Cell 17, 260 (2015).

[67] A. Marusyk, V. Almendro, and K. Polyak, Intra-Tumour Heterogeneity: A Looking Glass for Cancer?, Nat. Rev. Cancer 12, 323 (2012).

[68] J. Toner, Birth, Death, and Flight: A Theory of Malthusian Flocks, Phys. Rev. Lett. 108, 088102 (2012).

[69] V. Almendro, A. Marusyk, and K. Polyak, Cellular Heterogeneity and Molecular Evolution in Cancer, Annu. Rev. Pathol. 8, 277 (2013).
[70] R. Hoefflin, B. Lahrmann, G. Warsow, D. Hübschmann, C. Spath, B. Walter, X. Chen, L. Hofer, S. Macher-Goeppinger, Y. Tolstov et al., Spatial Niche Formation but Not Malignant Progression Is a Driving Force for Intratumoural Heterogeneity, Nat. Commun. 7, 11845 (2016).

[71] J. Lee, A. A. Abdeen, J. Hedhli, K. L. Wycislo, I. T. Dobrucki, T. M. Fan, L. W. Dobrucki, and K. A. Kilian, Melanoma Topology Reveals a Stem-Like Phenotype That Promotes Angiogenesis, Sci. Adv. 3, e1701350 (2017).

[72] D. S. Dean, Langevin Equation for the Density of a System of Interacting Langevin Processes, J. Phys. A 29, L613 (1996).

[73] M. Doi, Second Quantization Representation for Classical Many-Particle System, J. Phys. A 9, 1465 (1976).

[74] L. Peliti, Path Integral Approach to Birth-Death Processes on a Lattice, J. Phys. (Paris) 46, 1469 (1985).

[75] A. Gelimson and R. Golestanian, Collective Dynamics of Dividing Chemotactic Cells, Phys. Rev. Lett. 114, 028101 (2015).

[76] H. S. Samanta and J. K. Bhattacharjee, Nonequilibrium Statistical Physics with Fictitious Time, Phys. Rev. E 73, 046125 (2006).

[77] H. S. Samanta, J. K. Bhattacharjee, and D. Gangopadhyay, Growth Models and Models of Turbulence: A Stochastic Quantization Perspective, Phys. Lett. A 353, 113 (2006). 